\title{
Protein kinase B controls Mycobacterium tuberculosis growth via phosphorylation of the global transcriptional regulator Lsr2
}

Kawther Alqaseer ${ }^{1,2 \$}$, Obolbek Turapov ${ }^{1 \$}$, Philippe Barthe ${ }^{3}$, Heena Jagatia ${ }^{4}$, Angélique De Visch $^{3}$, Christian Roumestand $^{3}$, Malgorzata Wegrzyn $^{5}$, lona L. Bartek ${ }^{6}$, Martin I. Voskuil ${ }^{6}$, Helen O'Hare ${ }^{1,8}$, Adam A. Witney ${ }^{7}$, Martin Cohen-Gonsaud ${ }^{3^{*}}$, Simon J. Waddell ${ }^{4^{*}}$, Galina V. Mukamolova $^{1 *}$

${ }^{1}$ Leicester Tuberculosis Research Group, Department of Respiratory Sciences, University of Leicester, Leicester, LE2 9HN, UK; ${ }^{2}$ Department of Basic Science, Faculty of Nursing, University of Kufa, P.O. Box 21, Kufa, Najaf Governorate, Najaf, Iraq; ${ }^{3}$ Centre de Biochimie Structurale, CNRS, INSERM, University of Montpellier, 34090 Montpellier, France;

${ }^{4}$ Wellcome Trust Brighton and Sussex Centre for Global Health Research, Brighton and Sussex Medical School, University of Sussex, Brighton, BN1 9PX, UK; ${ }^{5}$ Core Biotechnology Services, University of Leicester, University Road, Leicester, LE1 7RH, UK; ${ }^{6}$ Department of Immunology and Microbiology, University of Colorado School of Medicine, Aurora, CO 80045, USA; ${ }^{7}$ Institute for Infection and Immunity, St George's University of London, London,

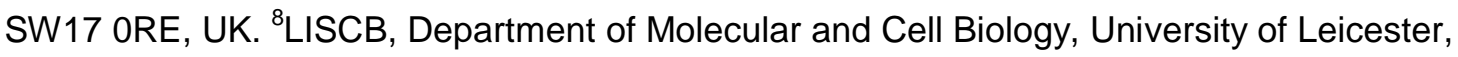
University Road, Leicester, LE1 7RH, UK.

\$Contributed equally to this work

*To whom correspondence should be addressed. E-mail: martin@cbs.cnrs.fr; s.waddell@bsms.ac.uk; gvm4@leicester.ac.uk. 


\section{ABSTRACT}

Mycobacterium tuberculosis is able to persist in the body through months of multi-drug therapy. Mycobacteria possess a wide range of regulatory proteins, including the essential protein kinase $B(P k n B)$, that control transitions between growth states. Here, we establish that depletion of PknB in replicating M. tuberculosis results in transcriptional adaptations that implicate the DNA-binding protein Lsr2 in coordinating these changes. We show that Lsr2 is phosphorylated by PknB, and that phosphorylation of Lsr2 at threonine 112 is important for M. tuberculosis growth and survival under hypoxic conditions. Fluorescence anisotropy and electrophoretic mobility shift assays demonstrate that phosphorylation reduces Lsr2 binding to DNA, and ChIP-sequencing confirms increased DNA binding of a phosphoablative (T112A) Lsr2 mutant in M. tuberculosis. Altered expression of target genes in T112A Lsr2 compared to wild type Lsr2 M. tuberculosis offers further evidence that phosphorylation mediates expression of the Lsr2 regulon. Structural studies reveal increased dynamics of the Lsr2 DNA binding domain from a T112D phosphomimetic Lsr2 mutant, providing a molecular basis for decreased DNA binding by phosphorylated Lsr2. Our findings suggest that, the essential protein kinase, PknB controls $M$. tuberculosis growth and adaptations to the changing host environment by phosphorylating the global transcriptional regulator Lsr2.

\section{INTRODUCTION}

Mycobacterium tuberculosis is a slow-growing bacterium that can replicate in humans and cause tuberculosis. The pathogen is able to rapidly shut-down its growth to persist in nonreplicating states in infected individuals, which can be modelled in the laboratory ${ }^{1} . M$. tuberculosis adaptation to stressful conditions is accompanied by dramatic changes in global protein phosphorylation but the importance of these modifications is poorly defined ${ }^{2} . M$. tuberculosis has eleven serine/threonine protein kinases which play significant roles in growth, virulence and metabolism ${ }^{3}$. In particular, protein kinase $\mathrm{B}(\mathrm{PknB})$ is indispensable for M. tuberculosis growth due to its critical role in the regulation of peptidoglycan biosynthesis ${ }^{4,5}$. It is also important for M. tuberculosis survival in hypoxic conditions and 
resuscitation during reaeration ${ }^{6}$. However, the molecular mechanism for PknB-mediated adaptation to hypoxia is unknown.

We have recently shown that PknB-depleted $M$. tuberculosis can grow in a sucrose magnesium medium $(\mathrm{SMM})^{5}$. Comparative phosphoproteomics of PknB-producing and PknB-depleted mycobacteria revealed substantial changes in profiles of phosphorylated proteins. Specifically, the transcriptional regulators Lsr2 and EthR showed increased phosphorylation in PknB-producing compared with PknB-depleted mycobacteria, indicating that these proteins may be PknB substrates. EthR is a TetR family transcriptional regulator that controls expression of EthA, a monoxygenase responsible for activation of the prodrug ethionamide ${ }^{7}$. Protein kinase $\mathrm{F}$ was found to phosphorylate EthR in vitro, which negatively regulated EthR binding to DNA ${ }^{8}$.

Lsr2 is a DNA binding protein that combines properties of a nucleoid associated protein ${ }^{9}$ and a global transcriptional regulator ${ }^{10}$. Lsr2 has over 1000 binding sites in $M$. tuberculosis $^{11,12}$. The precise role of Lsr2 in mycobacterial biology remains unclear, nevertheless parallels may be drawn with similar proteins from other bacteria. Lsr2 represents the first example of an H-NS-like protein identified outside Gram-negative bacteria, moreover Isr2 was able to complement the hns mutant in Escherichia coli ${ }^{13}$. Similar to the H-NS proteins, Lsr2 has been proposed to bind to the minor groove of DNA ${ }^{14}$ and to possess DNA bridging properties ${ }^{15}$. Deletion of $I s r 2$ in $M$. tuberculosis resulted in severe growth impairment on solid media, defects in persistence and adaptation to changing oxygen levels ${ }^{10}$. Additionally, Lsr2 has been shown to help protect DNA from reactive oxygen species, and over-expression of Lsr2 improved survival of mycobacteria treated with hydrogen peroxide ${ }^{16}$. However, deletion of Isr2 did not alter survival of $M$. tuberculosis exposed to hydrogen peroxide or mitomycin $\mathrm{C}^{10}$. Transcriptional profiling of an Isr2 deletion mutant revealed global changes involved in cell wall remodelling, respiration and lipid biosynthesis ${ }^{10}$.

Here we show that recombinant PknB phosphorylated Lsr2 at T112, a site that we identified previously by a proteomic approach, implying that $\mathrm{PknB}$ is directly responsible for 
controlling phosphorylation at this site. We demonstrate that phosphorylation of Lsr2 at threonine 112 is important for M. tuberculosis growth and adaptation to hypoxic conditions. Our results show that phosphorylation of Lsr2, or phosphomimetic T112D mutation, reduce Lsr2 binding to DNA, which is explained by increased dynamics in the DNA binding domain of phosphorylated Lsr2. Chromatin immunoprecipitation and transcriptomics further confirm that the T112A phosphoablative mutant of Lsr2 has an increased number of Lsr2-DNA binding events and modified expression of genes involved in $M$. tuberculosis stress responses and virulence. Based on our data, we propose that PknB-mediated phosphorylation controls Lsr2 binding to DNA in M. tuberculosis, providing a functional link between serine/threonine protein kinase signalling in replicating bacilli and regulatory networks that enable M. tuberculosis to survive dynamic environments during infection.

\section{RESULTS}

Transcriptome profiling of PknB-depleted M. tuberculosis reveals Lsr2-regulated gene expression signature

Two transcriptional regulators were significantly under-phosphorylated in PknB-depleted $M$. tuberculosis: Lsr2 and EthR ${ }^{5}$. To determine the resulting effect on gene expression, we compared the transcriptional profile of PknB-depleted versus PknB-producing $M$. tuberculosis, using the same pristinamycin-inducible PknB conditional mutant of $M$. tuberculosis (pknB-CM) ${ }^{17}$ in sucrose-magnesium medium (SMM) to support growth ${ }^{5}$. In total 99 genes were differentially expressed: 65 were induced and 34 repressed by PknBdepletion (Table S1). Two functional classes were identified in the genes overexpressed by PknB depletion: regulatory proteins and proteins involved in lipid metabolism. The predicted transcriptional regulators were csoR, rv1129c, rv1460, rv2017, rv2250c, rv3334, sigB, whiB3 and whiB6 (Table S1). These transcription factors have been shown to regulate copper homeostasis $(\mathrm{CsoR})^{18}$, iron-sulphur cluster biogenesis $(\mathrm{Rv} 1460)^{19}$, cholesterol catabolism $(\mathrm{Rv} 1129 \mathrm{c} / \operatorname{PrpR})^{20}$, the enduring hypoxic response $(\mathrm{Rv} 3334)^{21}$, multiple stress responses $(\mathrm{SigB})^{22}$, redox stress and complex lipid biosynthesis (WhiB3) ${ }^{23}$ and virulence factor 
expression (WhiB6) ${ }^{24}$. The transcriptional signature of PknB-depletion resembled features of intracellular growth, which significantly overlapped with $M$. tuberculosis macrophage-derived RNA profiles from several studies as reflected by hypergeometric probability values: $6.7 \times 10^{-}$ 23 25. $7.34 \times 10^{-1826} ; 3.57 \times 10^{-1727}$ (Fig. 1). This profile was exemplified by induction of pathways involved in mycobactin synthesis $(m b t B / C / D)$, complex lipid phthiocerol dimycocerosate (PDIM) biosynthesis ( $f a d D 26, p p s A / B / C / D)$, metabolism of alternative lipid carbon sources, the glyoxylate shunt $(i c)$, the methylcitrate cycle $(\operatorname{prpD} / C, \operatorname{prp} R)$ and triacylglycerol synthase (tgs 1). The isoniazid inducible genes (iniB/A/C), responsive to cell wall stress and cell wall targeting drugs were also upregulated ${ }^{28}$. Four of the nine genes coding for alternative ribosomal proteins, rpmB1, rpmB2, rpmG1, rps $N 2^{29}$ were induced by PknB depletion.

The 34 genes that were significantly repressed in PknB-depleted bacteria included pknB itself (6 fold down-regulated, as expected); $n u o A / B / C$, encoding subunits of NADH dehydrogenase I, which is part of the aerobic respiratory chain, and several genes involved in intermediary metabolism (Table S1). Overall these changes in replicating bacteria were comparable, in number of differentially expressed genes, to depletion of other regulators, for example DosR ${ }^{30}$. This is in contrast to the large-scale changes in gene expression after treatment with an inhibitor of PknB and PknA that would likely impact M. tuberculosis viability 31. In summary, PknB depletion in replicating bacteria resulted in co-ordinated changes to the transcriptome, suggesting that PknB may control the induction of alternative gene regulatory pathways.

Application of the Transcription Factor Over-Expression (TFOE) output tool ${ }^{32}$ predicted $^{2}$ $\mathrm{Rv} 0081^{33}$, DosR ${ }^{30}$ and $\mathrm{Lsr}^{10}$ as potential regulators of the observed gene expression patterns (Fig. 1). Rv0081 is a transcriptional hub which coordinates expression of over 50 transcription regulators controlling metabolic adaptation of M. tuberculosis ${ }^{33}$. However, to date there are no reports of Rv0081 regulation by phosphorylation. DosR has been shown to be phosphorylated by $\mathrm{PknH}^{34}$ but it was not under-phosphorylated during PknB depletion ${ }^{5}$. According to our data, PknB changed Lsr2 phosphorylation without impacting Lsr2 protein 
expression levels ${ }^{5}$, suggesting that the transcriptional changes between PknB-depleted and replete M. tuberculosis were likely caused by changes in PknB-mediated phosphorylation of Lsr2. We therefore investigated whether PknB could phosphorylate Lsr2 in vitro.

\section{PknB phosphorylates Lsr2 in vitro}

Purified Lsr2 was mixed with the recombinant kinase domain of $\mathrm{PknB}$ and incubated at $37^{\circ} \mathrm{C}$ with or without ATP; phosphorylation was detected using western blotting with an antiphosphothreonine antibody. Phosphorylated-Lsr2 was detected in the ATP-containing sample, demonstrating that PknB could indeed phosphorylate Lsr2 (Fig. 2A). Interestingly, phosphorylation resulted in a marked change in Lsr2 protein mobility on SDS-PAGE (Fig. 2B) and generated several bands, indicative of multiple phosphorylated isoforms. Massspectrometry confirmed the previously observed phosphosite on threonine $112^{5}$ and detected additional phosphorylations at threonine 8, threonine 22 and threonine 31 (Fig. 2C, D). The functional importance of these phosphorylation sites in Lsr2 was further investigated in complementation assays. We generated a panel of phosphoablative Lsr2 mutants in $M$. tuberculosis (Table S2) and investigated their phenotypes.

\section{Phosphorylation of Lsr2 on threonine 112 is necessary for M. tuberculosis growth}

Lsr2 deletion significantly impaired $M$. tuberculosis growth on solid media and the reintroduction of Isr2 on the pMV306 integrative plasmid $\left(\Delta / s r 2_{W T}\right)$ into the Isr2 deletion mutant fully complemented this defect (Fig. 3A \& Fig. S1), similarly to a previous study ${ }^{10}$. An Lsr2 deletion mutant containing the empty pMV306 plasmid $\left(\Delta / s r 2_{\mathrm{pMV} 306}\right)$ had the same growth pattern as the Isr2 deletion mutant (Fig. 3A), as expected. Lsr2 variants lacking the phosphorylation sites T8A, T22A and T31A individually restored bacterial growth and colony size to the same extent as wild type Lsr2 (Fig. S1). The T112A Lsr2 mutant $\left(\Delta / s r 2_{T 112 A}\right)$, however, failed to complement the $\Delta / s r 2$ phenotype (Fig. 3A, Fig. S1). Replacement of threonine 112 with an aspartate to mimic phosphorylation $\left(\Delta / s r 2_{\mathrm{T} 112 \mathrm{D}}\right)$ complemented the $\Delta /$ sr2 phenotype (Fig. 3A). 
We next investigated whether T112 mutations of Lsr2 influenced M. tuberculosis growth in liquid culture. As Fig. 3B demonstrates, $\Delta / s r 2_{\text {pMV} 306}$ and $\Delta / s r 2_{T 112 A}$ had significant growth defects compared with $\Delta / s r 2_{W T}$ and $\Delta / s r 2_{T 112 \mathrm{D}}$. Growth rates for $\Delta / s r 2_{\mathrm{pMV} 306}$ and $\Delta / s r 2_{\mathrm{T} 112 \mathrm{~A}}$ were $0.0157 \pm 0.03 \mathrm{~h}^{-1}$ and $0.0173 \pm 0.004 \mathrm{~h}^{-1}$, compared with $0.0414 \pm 0.004 \mathrm{~h}^{-1}$ and $0.0385 \pm 0.009 \mathrm{~h}^{-1}$ for $\Delta / s r 2_{\mathrm{WT}}$ and $\Delta / s r 2_{\mathrm{T} 112 \mathrm{D}}$. Thus, the T112A mutant failed to complement the growth defect of $\Delta / s r 2$ both in liquid and on solid media, suggesting that phosphorylation of Lsr2 at T112 is important for M. tuberculosis growth. Furthermore, western blot analysis using anti-Lsr2 antibody confirmed that wild type, T112A and T112D Lsr2 proteins were produced at similar levels, excluding the possibility that the $\Delta / s r 2_{T 112 \mathrm{~A}}$ phenotype was caused by altered Lsr2 expression (Fig. 3C). The phosphomimetic T112D Lsr2 had lower mobility in SDS PAGE, similar to phosphorylated Lsr2 (Fig. 3C and Fig. 2B).

\section{Lsr2 phosphorylation at threonine 112 is crucial for survival in hypoxic conditions but not in prolonged stationary phase}

We hypothesised that regulation of Lsr2 by PknB could account for the defects in survival of oxygen depletion seen when $p k n B$ and $/ s r 2$ were perturbed ${ }^{6,10}$. Thus, we assessed survival of M. tuberculosis carrying Lsr2 variants using the Wayne model of non-replicating persistence in hypoxia ${ }^{35}$. The viable counts of $\Delta / s r 2_{\text {pMV306 }}$ dropped dramatically after 7 weekincubation compared to $\Delta / s r 2_{\mathrm{WT}}$ and $\Delta / s r 2_{\mathrm{T} 112 \mathrm{D}}$ (Fig. 3D). Further incubation for 24 weeks resulted in near-complete loss of recoverable $\Delta / s r 2_{\text {pMV306 }}$ bacteria but did not significantly alter the survival of $\Delta / s r 2_{W T}$ and $\Delta / s r 2_{T 112 D}$. Interestingly, T112A Lsr2 could only partially complement the $\Delta / s r 2$ survival defect, suggesting a requirement for $\mathrm{T} 112$ phosphorylation in microaerobic conditions. After 24 week-incubation, viable counts of $\Delta / s r 2_{\mathrm{pMV} 306}$ and $\Delta / s r 2_{\mathrm{T} 112 \mathrm{~A}}$ were almost undetectable (Fig. 3D).

We also assessed the survival of $M$. tuberculosis expressing Lsr2 variants in late stationary phase of aerobically grown cultures by MPN and CFU counting. $4 / s r 2_{\text {pMV306 }}$ showed impaired survival compared to $\Delta / s r 2_{W T}$, resulting in a 1.5 order of magnitude 
difference in viable counts. In this model, the survival of T112A or T112D variants was not significantly different from wild type Lsr2 (Fig. S1B). Our results suggest that Lsr2 phosphorylation of T112 may be specifically required during adaptation to hypoxic conditions but not for survival in prolonged stationary phase.

Increased DNA binding in the T112A Lsr2 mutant of $M$. tuberculosis alters gene expression in M. tuberculosis

Phosphorylation of transcriptional regulators is known to influence their interaction with DNA ${ }^{8}$. Lsr2 binds at multiple sites in the M. tuberculosis genome ${ }^{14}$. We hypothesised that Lsr2 phosphorylation might influence Lsr2-DNA interactions resulting in differential binding patterns in phosphoablative Lsr2 compared to wild type Lsr2 M. tuberculosis. We used a custom anti-Lsr2 antibody to define genome-wide Lsr2 binding patterns in M. tuberculosis. In control experiments, no DNA fragments were precipitated from $\Delta / s r 2$ or $\Delta / s r 2_{\text {pMV306 }}$ mutants, confirming antibody specificity. We therefore determined the Lsr2 binding distribution from $\Delta / s r 2_{\mathrm{WT}}, \Delta / s r 2_{\mathrm{T} 112 \mathrm{~A}}$ and $\Delta / s r 2_{\mathrm{T} 112 \mathrm{D}}$ in logarithmic phase $M$. tuberculosis. An average of 690 Lsr2 binding fragments were identified for wild type Lsr2 from three biological replicates $\left(\Delta / s r 2_{W T}\right)$, with a median length of 413 bp (Fig. S2, Table S3). Lsr2 binding was identified throughout the M. tuberculosis chromosome, with binding peaks often at intergenic spaces or with binding running through several open reading frames. The binding pattern was wellconserved across biological replicates (Fig. 4A, Fig. S2, Table S3). The putative regulon of Lsr2, including genes with an Lsr2 binding site within or immediately upstream of the coding sequence, was defined as 1178 genes (Fig. 4A, Table S3). These genes, potentially directly regulated by Lsr2, are consistent with previously identified Lsr2 binding patterns ${ }^{14,36,12}$ (Fig. S2). Interestingly, gene expression signatures associated with inactivation of $\mathrm{Lsr} 2^{10}$, macrophage infection and acid-nitrosative stress were significantly enriched (hypergeometric probabilities $2.45 \times 10^{-27}, 3.02 \times 10^{-14}$ and $8.59 \times 10^{-15}$ respectively, Fig.S2), providing further evidence that Lsr2 may directly regulate gene expression. 
To establish whether phosphorylation changed the binding pattern of Lsr2 in $M$. tuberculosis, we compared phosphomimetic $\left(\Delta / s r 2_{T 112 \mathrm{D}}\right)$ and phosphoablative $\left(\Delta / s r 2_{\mathrm{T} 112 \mathrm{~A}}\right)$ to wild type Lsr2 $\left(\Delta / s r 2_{\mathrm{WT}}\right)$. There were 226 binding events with significantly greater abundance in $\Delta / s r 2_{T 112 A}$ in comparison to $\Delta / s r 2_{W T} M$. tuberculosis (Fig. S2, Fig. $4 \mathrm{~A}$ ). This potentially affected the expression of 94 genes (Table S4), including pathways involved in cell wall biosynthesis, lipid metabolism, PE/PPE protein synthesis and intermediary metabolism. No binding events were overrepresented in $\Delta / s r 2_{T_{112 D}}$ compared to $\Delta / s r 2_{\mathrm{WT}}$. Thus, our results suggest that while phosphomimetic Lsr2 $\left(\Delta / s r 2_{T 112 \mathrm{D}}\right)$ behaved similarly to wild type, phosphoablative Lsr2 $\left(\Delta / s r 2_{T 112 A}\right)$ bound DNA to a greater extent.

To probe whether altered binding of Lsr2 variants influenced gene expression we measured the expression of a panel of genes with predicted Lsr2 binding sites in $\Delta / s r 2_{p M V 306}$, $\Delta / s r 2_{\mathrm{WT}}$ and $\Delta / s r 2_{\mathrm{T} 112 \mathrm{~A}}$ genetic backgrounds. These genes included leuS, rpfA, rpfC sigA, and sodA. According to previously published data $\operatorname{rpf} A, \operatorname{rpfC}$ and $\operatorname{sod} A$ were significantly downregulated in the Isr2 deletion mutant ${ }^{10}$, while expression of $\operatorname{sod} A$ and leuS were predicted to be differentially regulated in $\Delta / s r 2_{\mathrm{T} 112 \mathrm{~A}}$ in our ChIP-Seq analysis. Lastly, sigA encoding a housekeeping sigma factor, has a putative Lsr2 binding site in the promoter region ${ }^{36}$, which could influence its expression. Fig. 4B shows that 4 genes $r p f A, r p f C, \operatorname{sig} A$ and $\operatorname{sod} A$ were indeed up-regulated in $\Delta / s r 2_{W T}$ compared with $\Delta / s r 2_{p M V 306}$. However, only expression levels of leuS and sodA, with divergent Lsr2 binding events, were statistically significantly different in $\Delta / s r 2_{T 112 A}$ compared with $\Delta / s r 2_{W T}$. Hence, our results confirm that the phosphorylation state of Lsr2 may influence gene expression patterns.

\section{PknB-mediated phosphorylation and T112D mutation reduce Lsr2 binding to DNA in vitro}

We further investigated the impact of phosphorylation on Lsr2 binding to DNA in vitro using Electrophoretic Mobility Shift Assays (EMSA) with a range of DNA fragments containing putative Lsr2 binding sites. Lsr2 was able to shift all DNA fragments when added at concentrations above $1.9 \mu \mathrm{M}$; there was no significant difference in binding patterns 
between the DNA fragments (Fig. S3). For example, previously published data ${ }^{36}$ indicated a putative Lsr2 binding site in the leuS coding region (5'-AATTCGGCAAAATCGGTAAG-3' marked with an asterisk in Fig. 4A); this fragment was shifted in EMSA by Lsr2 (Fig. 5A). A DNA fragment containing a mutated Lsr2 binding site where the central AT rich region was replaced with a GC-rich sequence (5'-AACTCGGCGAGGTCGGTCAG-3') was also shifted by Lsr2 (Fig. 5B). We tested other DNA fragments and all were shifted by Lsr2 regardless of their sequence, demonstrating that Lsr2 can bind non-specifically to DNA as previously suggested $^{28}$ and in agreement with our ChIP-Seq analysis.

We next investigated the effect of Lsr2 phosphorylation on binding to the leuS site. As shown in Fig. 5C, phosphorylation of Lsr2 by PknB completely abolished binding (Fig. 5C). Similar results were obtained with other DNA fragments (Fig. S3). This leuS observation correlates with our ChIP-Seq finding (Fig. 4A) of a greater number of leuS binding events in phosphoablative compared to wild type Lsr2. Lsr2 T112D showed reduced DNA binding in EMSA experiments (Fig. 5D), suggesting that T112D mimicked phosphorylated Lsr2 to some extent but still showed weak binding to the fragments. Thus, full-length Lsr2 could bind DNA non-specifically and phosphorylation or T112D mutation abolished or reduced this binding in vitro. In H-NS proteins the oligomerisation domain is important for shaping the nucleoid, while the DNA binding domain regulates gene expression ${ }^{37}$. We therefore investigated whether phosphorylation of the DNA binding domain (Lsr2 $\mathrm{BD}$ ) influenced binding to DNA. The truncated protein Lsr2 $\mathrm{BD}$ was not suitable for EMSA, so we used a fluorescence anisotropy approach.

Previous studies demonstrated that $\mathrm{Lsr}_{\mathrm{BD}}$ mainly recognised AT-rich DNA sequences that formed a hook-like structure ${ }^{14}$. We measured $\mathrm{Lsr}_{\mathrm{BD}}$ and $\mathrm{Lsr}_{\mathrm{BD}} \mathrm{T} 112 \mathrm{D}$ binding to an Alexa 488N labelled AT-rich DNA fragment (5'-CGCGCATATATGCG-3'), previously identified by Gordon and colleagues ${ }^{14}$ (Fig. 5E). At pH 7.5 the Kd value for Lsr2 $_{\mathrm{BD}}$ was $0.21 \pm 0.06 \mu \mathrm{M}$, while the $\mathrm{Kd}$ value for $\mathrm{Lsr}_{\mathrm{BD}} \mathrm{T} 112 \mathrm{~A}$ was $0.02 \pm 0.008 \mu \mathrm{M}$ and the Lsr2 $_{\mathrm{BD}} \mathrm{T} 112 \mathrm{D}$ showed no significant DNA binding (Kd could not be determined). Next, we tested binding of Lsr2 ${ }_{\mathrm{BD}}$ to the wild type and mutated leuS sequences where the central $\mathrm{AT}$ 
rich region was substituted by a GC-rich sequence (as described in the previous section). The binding of $L s r 2_{B D}$ to the leuS sequence was characterized with a $K d$ of $0.4 \pm 0.12 \mu \mathrm{M}$ (Fig. 5 F). No binding was observed for Lsr2 $\mathrm{BD}$ to the GC-mutated sequence (Fig. 5G). Lsr2 ${ }_{\mathrm{BD}} \mathrm{T} 112 \mathrm{D}$ did not bind to either leuS sequences (Fig. 5F-G). These results show that T112D mutation, mimicking the Lsr2 phosphorylated state, reduced Lsr2 $\mathrm{BD} T 112 \mathrm{D}$ binding to DNA in vitro. Hence, phosphorylation controls both Lsr2 and Lsr2 $\mathrm{BD}$ binding to DNA.

\section{Phosphomimetic T112D mutation changes conformation entropy of the Lsr2 DNA binding domain}

To elucidate a molecular basis for the reduced binding of phosphomimetic Lsr2 to DNA we compared nuclear magnetic resonance (NMR) structures of Lsr2 ${ }_{\mathrm{BD}} \mathrm{WT}$ and $\mathrm{Lsr}_{\mathrm{BD}} \mathrm{T} 112 \mathrm{D}$. In accordance with the previously published structure ${ }^{11}$, our data confirmed that Lsr2 $\mathrm{BD}$ consists of two perpendicular a-helices (a1, residues 78-89; a2, residues 102-112) linked by a long loop (residues 90-101), and this organisation was preserved in Lsr2 ${ }_{\mathrm{BD}} \mathrm{T} 112 \mathrm{D}$ (Table S5). However, T112D mutation resulted in a shorter a2 helix (Fig. S4), which ended with alanine 111 in Lsr2 $2_{\mathrm{BD}}$ T112D compared with threonine 112 in Lsr2 ${ }_{\mathrm{BD}} \mathrm{WT}$ (Fig. 6A and 6B). Our data suggest that both proteins were in monomeric states and the $\mathrm{N}$-terminal segment (residues 66-75) upstream of the DNA binding domain was disordered. We also found that in Lsr2 $2_{\mathrm{BD}}$ the methyl group of threonine 112 interacted with the tyrosine 108 and the hydroxyl group interacted with tryptophan 86 (Fig. 6C). These interactions did not form in the $\mathrm{Lsr}_{\mathrm{BD}} \mathrm{T} 112 \mathrm{D}$; however, they are unlikely to be important for DNA binding since $\mathrm{T} 112 \mathrm{~A}$ was able to bind DNA in our anisotropy experiments (Fig. 5E).

${ }^{15} \mathrm{~N}$ heteronuclear NMR relaxation analysis was performed to assess the dynamic behaviour of the two proteins (Fig. S5). In both isoforms two a helices showed similar amplitudes for internal motion. However, in Lsr2 $\mathrm{BD}$ mainly the C-terminal helix was affected, while in $\mathrm{Lsr}_{\mathrm{BD}} \mathrm{T} 112 \mathrm{D}$ the internal motion was extended to the $\mathrm{N}$-terminal helix. A previous study on the catabolite activator protein (CAP) demonstrated that different protein mutants with the same structure of interaction interface displayed very different affinity for their target 
$D N A^{38}$. The authors showed that changes of the binding affinity were linked to fast internal dynamics (conformation entropy). Similarly, the T112D mutation and, presumably, phosphorylation of threonine 112, resulted in a shorter a2 helix and a more mobile loop which increased the $\mathrm{Lsr}_{\mathrm{BD}}$ dynamics and impaired DNA binding.

\section{DISCUSSION}

M. tuberculosis can subvert the immune system to survive in the host for many years. This remarkable ability is determined by mechanisms that allow $M$. tuberculosis to respond to multiple environments and adjust metabolic activity and cell division. One of these mechanisms is protein phosphorylation involving eleven serine/threonine kinases, including protein kinase B. PknB is expressed during active replication and is essential for growth in standard media ${ }^{17}$. PknB has been implicated in the regulation of peptidoglycan biosynthesis ${ }^{39}$; its main substrate stimulates biosynthesis of peptidoglycan precursors ${ }^{4}$ and may facilitate their transport to the cell surface ${ }^{5}$. This regulation is essential for adjusting bacterial growth and synthesis of the cell wall. The external PASTA domain of PknB ${ }^{40,41}$ is essential for PknB-mediated signalling and its disruption results in bacterial death and alteration of antimicrobial susceptibility ${ }^{42,5,31}$. Here we present data demonstrating that PknB also controls global gene expression via another substrate, the DNA-binding protein Lsr2. Transcriptomic analysis of PknB-depleted M. tuberculosis suggest that PknB may silence alternative pathways, which are not important for growth but may be critical for mediating stress responses and virulence. These include enzymes involved in alternative metabolic pathways, synthesis of complex lipids, regulators of stress responses and antimicrobial tolerance (Table S1).

Here we demonstrate that Lsr2 could be phosphorylated by PknB on several threonines but only the T112 was essential for growth and survival of $M$. tuberculosis. The phosphoablative T112A mutant could not complement the growth defect of the Isr2 deletion mutant and was impaired in the Wayne hypoxia model. To investigate the molecular mechanism responsible for the growth defect in $\Delta / s r 2_{\mathrm{T} 112 \mathrm{~A}}$ we conducted ChIP-Seq analysis 
and compared DNA binding patterns in $\Delta / s r 2_{T 112 \mathrm{~A}}, \Delta / s r 2_{\mathrm{T} 112 \mathrm{D}}$ and $\Delta / s r 2_{\mathrm{WT}}$. In agreement with previously published data we detected multiple occurrences of DNA binding in all three Lsr2 backgrounds supporting both nucleoid shaping and gene regulatory functions of Lsr2. However, phosphoablative Lsr2 mutation T112A resulted in increased DNA binding, potentially directly affecting expression of 94 genes. Interestingly, most of these genes were not essential for growth, moreover deletion of some of these genes has been previously shown to be advantageous for growth ${ }^{43}$. The latter included genes of unknown function (Rv0888, Rv1958, Rv1957), PE/PPE genes (Rv0878, Rv1983), aprA, and genes controlling transport of PDIM ( $d r r B$ and $d r r C$ ). While products of these genes might be disadvantageous for growth in vitro, they likely play a critical role in adaptations to stress and virulence ${ }^{27,44,45}$.

We next investigated how phosphorylation affected Lsr2 binding to DNA. Our data demonstrate that PknB phosphorylation of Lsr2 in vitro completely abolished DNA binding, while the phosphomimetic mutation reduced Lsr2-DNA interaction. We have not investigated the effect of T8, T22 and T31 phosphorylations on DNA binding. Based on previously published data we hypothesise that phosphorylation of these sites in the oligomerisation domain might be important for controlling nucleoid shape and DNA bridging properties ${ }^{37}$. Our data suggest that phosphorylation of T112 in the DNA binding domain controls binding of Lsr2 to AT-rich DNA sequences which leads to alteration of gene expression. Our structural studies further confirm that phosphomimetic T112D mutation results in a shorter C-terminal helix and increased dynamics of the DNA binding domain, leading to impaired Lsr2-DNA binding.

Post-translational modifications are common mechanisms for regulation of DNA binding both in eukaryotes ${ }^{46}$ and prokaryotes ${ }^{47}$. H-NS protein, a homologue of Lsr2 in E. coli, has been shown to be acetylated ${ }^{48}$ and phosphorylated ${ }^{49}$, however the precise function of these modifications remains to be characterised. Our data show that phosphorylation of Lsr2 is important for $M$. tuberculosis growth and this may be a key mechanism for controlling mycobacterial adaptations to permissive and non-permissive environments. Thus, PknB 
mediates two critical components of mycobacterial growth, peptidoglycan biosynthesis and gene expression of alternative pathways.

Based on our data we propose that PknB controls $M$. tuberculosis growth by phosphorylating Lsr2. Like other H-NS-like proteins Lsr2 plays a dual role in mycobacterial biology, it shapes and protects the nucleoid and it controls gene expression ${ }^{9}$. However, unlike H-NS proteins in Gram-negative bacteria which mainly silence the expression of foreign DNA ${ }^{50,37}$ Lsr2 regulates expression of genes that are essential for growth, virulence and adaptation ${ }^{10}$. Our data suggest that phosphorylation of $\mathrm{T} 112$ might be important for tuning gene expression during growth and the dynamic change between phosphorylated and non-phosphorylated Lsr2 may help to adjust transcriptional patterns according to growth conditions (Fig. 7A). Reduced T112 phosphorylation, for example during starvation, may increase Lsr2 binding and up-regulate pathways which are critical for $M$. tuberculosis survival under these conditions (Fig. 7B).

While there are many outstanding questions on the precise mechanisms of PknBmediated regulation of gene expression and Lsr2 binding to DNA, our findings provide a functional link between serine/threonine protein kinase signalling and transcriptional regulatory pathways that enable $M$. tuberculosis to survive the varied environments encountered during infection.

\section{METHODS}

\section{Organisms and media}

Mycobacterium tuberculosis H37Rv was grown in Middlebrook 7H9 (Becton, Dickinson and Company) liquid media supplemented with $10 \%$ (v/v) Albumin-Dextrose Complex (ADC), $0.2 \%(\mathrm{v} / \mathrm{v})$ glycerol and $0.1 \%(\mathrm{w} / \mathrm{v})$ Tween 80 at $37^{\circ} \mathrm{C}$ with shaking. The M. tuberculosis H37Rv PknB conditional mutant ( $p k n B-C M)$ was grown in sucrose magnesium medium (SMM) containing hygromycin with or without pristinamycin ${ }^{5}$. SMM comprised of $0.3 \mathrm{M}$ sucrose, $20 \mathrm{mM} \mathrm{MgSO}_{4}, 0.1 \%$ Tween 80 (w/v), 10\% (v/v) ADC in standard $7 \mathrm{H} 9$ broth. Bacterial growth was measured by absorbance at $580 \mathrm{~nm}$, or by colony-forming unit (CFU) 
counting on 7H10 agar (Becton, Dickinson and Company), or by Most-Probable Number (NPN) counting using established protocols ${ }^{51}$; MPN counts were calculated using the MPN calculator program ${ }^{52}$. Escherichia coli OverExpress ${ }^{\mathrm{TM}} \mathrm{C} 41$ (DE3) and $\mathrm{DH} 5 \alpha$ were grown in Lysogeny broth (LB). Antibiotics were used at the following concentrations $(\mu \mathrm{g} / \mathrm{ml})$ : pristinamycin, 0.5; kanamycin, 50; hygromycin, 50; ampicillin, 50.

\section{Generation Lsr2 complementation mutants}

The Rv3597 (Isr2) coding sequence with additional 200 bp upstream region was amplified from the M. tuberculosis H37Rv genome using primers Isr2-pMV306F and Isr2-pMV306R and cloned into the integrative plasmid pMV306. Lsr2 M. tuberculosis variants were obtained using the GeneArt ${ }^{\mathrm{TM}}$ Site-Directed Mutagenesis System (Thermo Fisher Scientific) according to the manufacturer's instructions. For generation of T112A and T112D mutants Isr2-pMV306RA and Isr2-pMV306RD primers were used. All constructs were sequenced before transformation into the previously described Isr2 deletion mutant ${ }^{10}$. Bacterial strains generated in this study are shown in Table S2.

\section{Wayne model of non-replicating persistence}

The model was setup as previously described ${ }^{35}$. CFU and MPN counts were determined at 0, 7 and 12-week time points. Statistical analysis was performed using one-way ANOVA (GraphPad Prism) where $\Delta / s r 2_{\mathrm{pMV}}$ or $\Delta / s r 2_{\mathrm{T} 112 \mathrm{~A}}$ data were compared to $\Delta / s r 2_{\mathrm{WT}}$ and $\Delta / s r 2_{\mathrm{T} 112 \mathrm{D}}$ data.

\section{Transcriptomic analyses}

M. tuberculosis $\mathrm{H} 37 \mathrm{Rv} \mathrm{PknB}$ conditional mutant (pknB-CM) was grown to $\mathrm{OD}_{580 \mathrm{~nm}} \sim 0.7$ in SMM with or without pristinamycin. M. tuberculosis RNA was extracted from three biological replicates using the GTC/Trizol method ${ }^{53}$. The RNA samples were purified and DNasetreated using RNeasy columns (Qiagen). M. tuberculosis RNA $(2 \mu \mathrm{g})$ was enzymaticallylabelled with Сy3 fluorophore and hybridised to a M. tuberculosis complex microarray (ArrayExpress accession number A-BUGS-41) as previously described ${ }^{54}$. Significantly differentially expressed genes (Table S1) were identified using a moderated t-test ( $p$-value 
$<0.05$ with Benjamini and Hochberg multiple testing correction), and fold change $>1.8$ in GeneSpring 14.5 (Agilent Technologies). Hypergeometric probability and TFOE analysis ${ }^{32}$ was used to identify significantly enriched signatures.

For quantitative RT-PCR, total RNA was isolated from triplicate M. tuberculosis cultures of $\Delta / s r 2_{\mathrm{T} 112 \mathrm{~A}}, \Delta / s r 2_{\mathrm{T} 112 \mathrm{D}}, \Delta / s r 2_{\mathrm{WT}}$ and $\Delta / s r 2_{\mathrm{pMV}}$ as described above and cDNA generated using Superscript Reverse Transcriptase II and mycobacterial genome-directed primers ${ }^{55}$. Q-PCR was performed in a Corbett Rotor Gene 6000 real time thermocycler using Absolute QPCR SYBR Green mix and gene expression values were normalised to 16S rRNA expression.

Statistical analysis was performed by one-way ANOVA where $4 / s r 2_{W T}$ was compared to $\Delta / s r 2_{T 112 \mathrm{~A}}$ and $\Delta / s r 2_{\mathrm{T} 112 \mathrm{D}}$.

\section{ChIP-Seq analysis}

DNA-Lsr2 interactions in $\Delta / s r 2_{\mathrm{T} 112 \mathrm{~A}}, \Delta / s r 2_{\mathrm{T} 112 \mathrm{D}}, \Delta / s r 2_{\mathrm{WT}}$ were assayed using methods adapted from Minch et al' ${ }^{12}$. Briefly, mid log-phase M. tuberculosis H37Rv cultures $\left(\mathrm{OD}_{600}\right.$ 0.4-0.6) were crosslinked with $1 \%$ formaldehyde, followed by incubation with $125 \mathrm{mM}$ glycine for 5 minutes at $37^{\circ} \mathrm{C}$. The cells were mechanically lysed and then sonicated to produce $200-500$ bp fragments. Input control samples were taken for each genotype before antibody was added to assess antibody specificity. Samples were immunoprecipitated using a polyclonal anti-rabbit anti-Lsr2 antibody and protein-G agarose beads. The Lsr2 complexes were decrosslinked by heating at $65^{\circ} \mathrm{C}$ overnight and proteins removed by treatment with proteinase $\mathrm{K}(10 \mathrm{mg} / \mathrm{ml})$ for $2 \mathrm{~h}$ at $55^{\circ} \mathrm{C}$. The DNA samples were column-purified (Qiagen) and the quality of purified IP-Lsr2 DNA verified using the Qubit DNA HS quantification assay and Nanodrop spectrophotometer. Libraries were prepared and sequenced using Illumina HiSeq SE50, 20 million reads (Novogene, Hong Kong). Raw fastq files were aligned to the $M$. tuberculosis H37Rv (NC_000962.3) reference genome using bwa samse ${ }^{56}$. MACS2 (version 2.1.1.20160309) ${ }^{57}$ was used to compare each of the input controls to the immunoprecipitated samples, identifying Lsr2 binding sites (callpeak) using default parameters but including "-g 
4.41e+06 -nomodel -extsize 147" (Table S3). Differential peaks comparing $\Delta / s r 2_{\mathrm{T} 112 \mathrm{~A}}$ to $\Delta / s r{ }_{W T}$ were then identified using "macs2 bdgdiff".

\section{Generation and purification of recombinant proteins}

The M. tuberculosis H37Rv Isr2 coding sequence was amplified using Platinum Taq-HF polymerase (primers in Table S6) and cloned into pET15-TEV. After confirmation by sequencing, the constructs were transformed into E. coli OverExpress ${ }^{\mathrm{TM}}$ C41 (DE3) competent cells (strain details are summarised in Table S2).

For protein expression, E. coli cultures were grown in LB with ampicillin to mid-log phase $\left(\mathrm{OD}_{600} 0.6-0.8\right)$ at $37^{\circ} \mathrm{C}$ with shaking at $200 \mathrm{rpm}$ before adding $0.5 \mathrm{mM}$ isopropyl $\beta-\mathrm{D}-1$ thiogalactopyranoside followed by incubation at $18^{\circ} \mathrm{C}$ overnight. The recombinant Lsr2 proteins were purified using immobilized-metal affinity chromatography (Ni-NTA agarose, Qiagen) and size exclusion chromatography. The recombinant catalytic domain of PknB was purified using Glutathione Sepharose 4B GST-tagged protein purification resin (GE Healthcare) as previously described ${ }^{5}$.

\section{Lsr2 phosphorylation in vitro}

Purified recombinant Lsr2 $(10 \mu \mathrm{M})$ was mixed with the recombinant catalytic domain of PknB $(5 \mu \mathrm{M})$ in a kinase buffer (20 mM Tris- $\mathrm{HCl}, \mathrm{pH} 8.0 ; 0.5 \mathrm{mM}$ DTT; $10 \mathrm{mM} \mathrm{MgCl}$; $0.2 \mathrm{mM}$ ATP) and incubated at $37^{\circ} \mathrm{C}$ for $1 \mathrm{~h}$. Phosphorylation was confirmed by western blot analysis using a phospho-threonine antibody (Cell Signaling Technology). Phosphorylated residues were identified in trypsin-digested proteins using a LTQ-Orbitrap-Velos mass spectrometer.

\section{Protein electrophoresis and western blotting}

Proteins were separated on $4-20 \%$ gradient SERVA gels and transferred onto a nitrocellulose membrane using a Trans-Blot ${ }^{\circledR}$ Turbo $^{\mathrm{TM}}$ Transfer System (Bio-Rad). SIGMAFASTTM $B C I P \circledast / N B T$ or SignalFire ${ }^{\mathrm{TM}}$ Elite ECL Reagent were used to visualize proteins on C-DiGit Chemiluminescent Blot Scanner (LI-COR Biosciences). The following antibodies were used: custom polyclonal antibody raised against Lsr2 in rabbit (Gemini Biosciences); monoclonal murine anti-poly-histidine antibody (Sigma-Aldrich); phospho- 
threonine antibody (Cell Signaling Technology); monoclonal anti-M. tuberculosis GroEL2 (Rv0440), clone IT-70 (BEIResources); mouse anti-rabbit lgG antibody:alkaline phosphatase (Sigma-Aldrich; anti-mouse IgG (whole molecule:alkaline phosphatase antibody produced in rabbit (Sigma-Aldrich), and anti-rabbit IgG, HRP-linked antibody (Cell Signaling Technology).

\section{Electrophoretic mobility shift assay (EMSA)}

EMSAs were carried out with DNA fragments amplified by PCR or annealed nucleotides (Table S6) as described previously ${ }^{15}$. Briefly, DNA (1.2 nM) was mixed with indicated amounts of Lsr2 in a total volume of $20 \mu \mathrm{l}$ reaction buffer containing $(10 \mathrm{mM}$ Tris- $\mathrm{HCl}, \mathrm{pH}$ 7.5, $50 \mathrm{mM} \mathrm{KCl}, 1 \mathrm{mM} \mathrm{DTT}, 5 \mathrm{mM} \mathrm{MgCl}_{2}$, and 2.5\% glycerol). The mixture was incubated for $30 \mathrm{~min}$ at room temperature followed by native polyacrylamide gel electrophoresis using $8 \%$ gels in $0.5 \times$ Tris-Borate-EDTA buffer, $\mathrm{pH} 7.5$ for $24 \mathrm{~min}$ at $120 \mathrm{~V}$. The gels were stained with SYBR Safe DNA stain (Thermo Fisher Scientific) and visualised using a ChemiDoc system (Bio-Rad).

\section{Fluorescence anisotropy}

Custom made 5' Alexa Fluor 488 succinimidyl ester labelled oligonucleotide probe (sequence 5'-CGCATATATGCGCG-3) was purchased from Integrated DNA Technologies. Steady-state fluorescence anisotropy binding titrations were performed on a Tecan Saphire II microplate reader, using a 470nm LED for excitation and a monochromator set at 530nm (bandwidth 20nm) for emission.

\section{Determination of solution structures and dynamics of $\mathrm{Lsr}_{\mathrm{BD}}$ and $\mathrm{Lsr} 2_{\mathrm{BD}} \mathrm{T} 112 \mathrm{D}$}

All ${ }^{1} \mathrm{H}^{15} \mathrm{~N}$ double-resonance NMR experiments were performed at $20^{\circ} \mathrm{C}$ on Bruker Avance III spectrometers (700 or $800 \mathrm{MHz}$ ). NMR samples of $0.5 \mathrm{mM}{ }^{15} \mathrm{~N}$-labeled protein dissolved in $25 \mathrm{mM}$ sodium phosphate buffer $(\mathrm{pH} 6.8), 150 \mathrm{mM} \mathrm{NaCl}$ with $10 \% \mathrm{D}_{2} \mathrm{O}$ for the lock. ${ }^{1} \mathrm{H}$ chemical shifts were directly referenced to the methyl resonance of DSS, while ${ }^{15} \mathrm{~N}$ chemical shifts were referenced indirectly to the absolute ${ }^{15} \mathrm{~N} /{ }^{1} \mathrm{H}$ frequency ratio. All NMR spectra were processed with GIFA ${ }^{58}$ and analysed with CINDY (Padilla et al., paper in preparation).

Chemical shift assignments were made using standard NOESY, TOCSY experiments performed on the ${ }^{15} \mathrm{~N}$-labeled protein sample. NOE cross-peaks identified on $3 \mathrm{D}\left[{ }^{1} \mathrm{H},{ }^{15} \mathrm{~N}\right]$ 
NOESY-HSQC (mixing time $160 \mathrm{~ms}$ ) were assigned through automated NMR structure calculations with CYANA $2.1^{59}$. Backbone $\phi$ and $\varphi$ torsion angle constraints were obtained from a database search procedure on the basis of backbone $\left({ }^{15} \mathrm{~N}, \mathrm{HN}, \mathrm{H \alpha}\right)$ chemical shifts using TALOS $+{ }^{60}$.

For each protein, a total of 200 three-dimensional structures were generated using the torsion angle dynamics protocol of CYANA 2.1. The 20 best structures of each protein (based on the final target penalty function values) were minimized with CNS 1.2. All statistical parameters are summarised in Table S5. Relaxation rate constant measurements were performed on a $0.5 \mathrm{mM}$ protein sample, at $18.8 \mathrm{~T}(800 \mathrm{MHz})$. The pulse sequences used to determine ${ }^{15} \mathrm{~N} R_{N}\left(N_{z}\right)\left(R_{1}\right), R_{N}\left(N_{x y}\right)\left(R_{2}\right)$, and ${ }^{15} N\left\{{ }^{1} H\right\}$ NOE values were similar to those described ${ }^{61}$. The ${ }^{15} \mathrm{~N}$ longitudinal relaxation rates $\left(\mathrm{R}_{\mathrm{N}}\left(\mathrm{N}_{\mathrm{z}}\right)\right)$ were obtained from 10 standard inversion-recovery experiments, with relaxation delays ranging from $18 \mathrm{~ms}$ to $1206 \mathrm{~ms}$. The ${ }^{15} \mathrm{~N}$ transverse relaxation experiments $\left(\mathrm{R}_{\mathrm{N}}\left(\mathrm{N}_{\mathrm{xy}}\right)\right)$ were obtained from 10 standard CPMG experiments, with relaxation delays ranging from $16 \mathrm{~ms}$ to $160 \mathrm{~ms}$. Both series of experiments were acquired in two single interleaved matrices to ensure uniformity of the experimental conditions. Heteronuclear ${ }^{15} \mathrm{~N}\left\{{ }^{1} \mathrm{H}\right\}$ NOE were determined from the ratio of two experiments, with and without saturation.

\section{Data availability}

Fully annotated microarray data have been deposited in ArrayExpress; accession number EMTAB-7627, https://www.ebi.ac.uk/arrayexpress.

ChIP-Seq datasets have been deposited in the European Nucleotide Archive (ENA); study accession number PRJEB31102, http://www.ebi.ac.uk/ena/data/view/PRJEB3110.

The coordinates for Lsr2 protein structures have been deposed in the PDB https://www.wwpdb.org; accession numbers PDB6QKP and PDB 6QKQ for Lsr2 ${ }_{\mathrm{BD}}$ and Lsr2 ${ }_{\mathrm{BD}} \mathrm{T} 112 \mathrm{D}$, respectively.

\section{REFERENCES}

1. Wayne, L.G. \& Sohaskey, C.D. Nonreplicating persistence of Mycobacterium 
tuberculosis. Annu Rev Microbiol 55, 139-163 (2001).

2. Prisic, S. et al. Extensive phosphorylation with overlapping specificity by

Mycobacterium tuberculosis serine/threonine protein kinases. Proc Natl Acad Sci U S

A 107, 7521-7526 (2010).

3. Prisic, S. \& Husson, R.N. Mycobacterium tuberculosis Serine/Threonine Protein

Kinases. Microbiol Spectr 2 (2014).

4. Boutte, C.C. et al. A cytoplasmic peptidoglycan amidase homologue controls

mycobacterial cell wall synthesis. Elife 5, e 14590 (2016).

5. Turapov, O. et al. Two faces of CwIM, an essential PknB substrate, in

Mycobacterium tuberculosis. Cell Reports 25, 57-67 (2018).

6. Ortega, C. et al. Mycobacterium tuberculosis Ser/Thr protein kinase B mediates an oxygen-dependent replication switch. PLoS Biol 12, e1001746 (2014).

7. Baulard, A.R. et al. Activation of the pro-drug ethionamide is regulated in mycobacteria. J Biol Chem 275, 28326-28331 (2000).

8. Leiba, J. et al. The Mycobacterium tuberculosis transcriptional repressor EthR is negatively regulated by Serine/Threonine phosphorylation. Biochem Biophys Res Commun 446, 1132-1138 (2014).

9. Kriel, N.L. et al. Mycobacterial nucleoid associated proteins: An added dimension in gene regulation. Tuberculosis (Edinb) 108, 169-177 (2018).

10. Bartek, I.L. et al. Mycobacterium tuberculosis Lsr2 is a global transcriptional regulator required for adaptation to changing oxygen levels and virulence. MBio 5, e0110601114 (2014).

11. Gordon, B.R. et al. Lsr2 is a nucleoid-associated protein that targets AT-rich sequences and virulence genes in Mycobacterium tuberculosis. Proc Natl Acad Sci U $S$ A 107, 5154-5159 (2010).

12. Minch, K.J. et al. The DNA-binding network of Mycobacterium tuberculosis. Nat Commun 6, 5829 (2015).

13. Gordon, B.R., Imperial, R., Wang, L., Navarre, W.W. \& Liu, J. Lsr2 of Mycobacterium 
represents a novel class of H-NS-like proteins. J Bacteriol 190, 7052-7059 (2008).

14. Gordon, B.R. et al. Structural basis for recognition of AT-rich DNA by unrelated xenogeneic silencing proteins. Proc Natl Acad Sci U S A 108, 10690-10695 (2011).

15. Chen, J.M. et al. Lsr2 of Mycobacterium tuberculosis is a DNA-bridging protein.

Nucleic Acids Res 36, 2123-2135 (2008).

16. Colangeli, R. et al. The multifunctional histone-like protein Lsr2 protects mycobacteria against reactive oxygen intermediates. Proc Natl Acad Sci U S A 106, 4414-4418 (2009).

17. Forti, F., Crosta, A. \& Ghisotti, D. Pristinamycin-inducible gene regulation in mycobacteria. J Biotechnol 140, 270-277 (2009).

18. Marcus, S.A., Sidiropoulos, S.W., Steinberg, H. \& Talaat, A.M. CsoR is essential for maintaining copper homeostasis in Mycobacterium tuberculosis. PLoS One 11, e0151816 (2016).

19. Willemse, D. et al. Rv1460, a SufR homologue, is a repressor of the suf operon in Mycobacterium tuberculosis. PLoS One 13, e0200145 (2018).

20. Masiewicz, P., Brzostek, A., Wolanski, M., Dziadek, J. \& Zakrzewska-Czerwinska, J. A novel role of the PrpR as a transcription factor involved in the regulation of methylcitrate pathway in Mycobacterium tuberculosis. PLoS One 7, e43651 (2012).

21. Rustad, T.R., Harrell, M.I., Liao, R. \& Sherman, D.R. The enduring hypoxic response of Mycobacterium tuberculosis. PLoS One 3, e1502 (2008).

22. Lee, J.H., Karakousis, P.C. \& Bishai, W.R. Roles of SigB and SigF in the Mycobacterium tuberculosis sigma factor network. J Bacteriol 190, 699-707 (2008).

23. Mehta, M. \& Singh, A. Mycobacterium tuberculosis WhiB3 maintains redox homeostasis and survival in response to reactive oxygen and nitrogen species. Free Radic Biol Med 131, 50-58 (2018).

24. Bosserman, R.E. et al. WhiB6 regulation of ESX-1 gene expression is controlled by a negative feedback loop in Mycobacterium marinum. Proc Natl Acad Sci U S A 114, E10772-E10781 (2017). 
25. Tailleux, L. et al. Probing host pathogen cross-talk by transcriptional profiling of both Mycobacterium tuberculosis and infected human dendritic cells and macrophages. PLoS One 3, e1403 (2008).

26. Schnappinger, D. et al. Transcriptional Adaptation of Mycobacterium tuberculosis within Macrophages: Insights into the Phagosomal Environment. J Exp Med 198, 693-704 (2003).

27. Rohde, K.H., Abramovitch, R.B. \& Russell, D.G. Mycobacterium tuberculosis invasion of macrophages: linking bacterial gene expression to environmental cues. Cell Host Microbe 2, 352-364 (2007).

28. Colangeli, R. et al. Transcriptional regulation of multi-drug tolerance and antibioticinduced responses by the histone-like protein Lsr2 in M. tuberculosis. PLoS Pathog 3, e87 (2007).

29. Prisic, S. et al. Zinc regulates a switch between primary and alternative S18 ribosomal proteins in Mycobacterium tuberculosis. Mol Microbiol 97, 263-280 (2015).

30. Park, H.D. et al. Rv3133c/dosR is a transcription factor that mediates the hypoxic response of Mycobacterium tuberculosis. Mol Microbiol 48, 833-843 (2003).

31. Carette, X. et al. Multisystem Analysis of Mycobacterium tuberculosis reveals kinasedependent remodeling of the pathogen-environment interface. MBio 9 (2018).

32. Rustad, T.R. et al. Mapping and manipulating the Mycobacterium tuberculosis transcriptome using a transcription factor overexpression-derived regulatory network. Genome Biol 15, 502 (2014).

33. Galagan, J.E. et al. The Mycobacterium tuberculosis regulatory network and hypoxia. Nature 499, 178-183 (2013).

34. Chao, J.D. et al. Convergence of Ser/Thr and two-component signaling to coordinate expression of the dormancy regulon in Mycobacterium tuberculosis. J Biol Chem 285, 29239-29246 (2010).

35. Wayne, L.G. \& Sramek, H.A. Metronidazole is bactericidal to dormant cells of Mycobacterium tuberculosis. Antimicrob Agents Chemother 38, 2054-2058 (1994). 
36. Reddy, T.B. et al. TB database: an integrated platform for tuberculosis research. Nucleic Acids Res 37, D499-508 (2009).

37. Winardhi, R.S., Yan, J. \& Kenney, L.J. H-NS Regulates gene expression and compacts the nucleoid: insights from single-molecule experiments. Biophys $J 109$, $1321-1329(2015)$

38. Tzeng, S.R. \& Kalodimos, C.G. Protein activity regulation by conformational entropy. Nature 488, 236-240 (2012).

39. Gee, C.L. et al. A phosphorylated pseudokinase complex controls cell wall synthesis in mycobacteria. Sci Signal 5, ra7 (2012).

40. Barthe, P., Mukamolova, G.V., Roumestand, C. \& Cohen-Gonsaud, M. The structure of PknB extracellular PASTA domain from Mycobacterium tuberculosis suggests a ligand-dependent kinase activation. Structure 18, 606-615 (2010).

41. Prigozhin, D.M. et al. Structural and genetic analyses of the Mycobacterium tuberculosis protein kinase B sensor domain identify a potential ligand-binding site. $J$ Biol Chem 291, 22961-22969 (2016).

42. Turapov, O. et al. The external PASTA domain of the essential serine/threonine protein kinase PknB regulates mycobacterial growth. Open Biol 5, 150025 (2015).

43. DeJesus, M.A. et al. Comprehensive essentiality analysis of the Mycobacterium tuberculosis genome via saturating transposon mutagenesis. MBio 8 (2017).

44. Camacho, L.R. et al. Analysis of the phthiocerol dimycocerosate locus of Mycobacterium tuberculosis. Evidence that this lipid is involved in the cell wall permeability barrier. J Biol Chem 276, 19845-19854 (2001).

45. Sassetti, C.M. \& Rubin, E.J. Genetic requirements for mycobacterial survival during infection. Proc Natl Acad Sci U S A 100, 12989-12994 (2003).

46. Bannister, A.J. \& Kouzarides, T. Regulation of chromatin by histone modifications. Cell Res 21, 381-395 (2011).

47. Dilweg, I.W. \& Dame, R.T. Post-translational modification of nucleoid-associated proteins: an extra layer of functional modulation in bacteria? Biochem Soc Trans 46, 
1381-1392 (2018).

48. Weinert, B.T. et al. Acetyl-phosphate is a critical determinant of lysine acetylation in E. coli. Mol Cell 51, 265-272 (2013).

49. Lin, M.H., Sugiyama, N. \& Ishihama, Y. Systematic profiling of the bacterial phosphoproteome reveals bacterium-specific features of phosphorylation. Sci Signal 8, rs10 (2015).

50. Navarre, W.W., McClelland, M., Libby, S.J. \& Fang, F.C. Silencing of xenogeneic DNA by H-NS-facilitation of lateral gene transfer in bacteria by a defense system that recognizes foreign DNA. Genes Dev 21, 1456-1471 (2007).

51. Loraine, J., Pu, F., Turapov, O. \& Mukamolova, G.V. Development of an in vitro assay for detection of drug-Induced Resuscitation-promoting-factor-dependent mycobacteria. Antimicrob Agents Chemother 60, 6227-6233 (2016).

52. Jarvis, B., Wilrich, C. \& Wilrich, P.T. Reconsideration of the derivation of Most Probable Numbers, their standard deviations, confidence bounds and rarity values. $J$ Appl Microbiol 109, 1660-1667 (2010).

53. Waddell, S.J.a.B., P.D. Use of DNA arrays to study transcriptional responses to antimycobacterial compounds. I. In Antibiotic Resistance Protocols (SH Gillespie and TD McHugh Eds.) Methods Mol Biol 642, 75-91 (2010).

54. Salina, E.G. et al. Potassium availability triggers Mycobacterium tuberculosis transition to, and resuscitation from, non-culturable (dormant) states. Open Biol 4 (2014).

55. Rachman, H., Lee, J.S., Angermann, J., Kowall, J. \& Kaufmann, S.H. Reliable amplification method for bacterial RNA. J Biotechnol 126, 61-68 (2006).

56. Li, H. \& Durbin, R. Fast and accurate short read alignment with Burrows-Wheeler transform. Bioinformatics 25, 1754-1760 (2009).

57. Zhang, Y. et al. Model-based analysis of ChIP-Seq (MACS). Genome Biol 9, R137 (2008).

58. Pons, J.L., Malliavin, T.E. \& Delsuc, M.A. Gifa V. 4: A complete package for NMR 
data set processing. J Biomol NMR 8, 445-452 (1996).

59. Guntert, P. Automated NMR structure calculation with CYANA. Methods Mol Biol 278, 353-378 (2004).

60. Shen, Y., Delaglio, F., Cornilescu, G. \& Bax, A. TALOS+: a hybrid method for predicting protein backbone torsion angles from NMR chemical shifts. J Biomol NMR 44, 213-223 (2009).

61. Barthe, P. et al. Refined solution structure and backbone dynamics of $15 \mathrm{~N}$-labeled C12A-p8MTCP1 studied by NMR relaxation. J Biomol NMR 15, 271-288 (1999).

62. Kapopoulou, A., Lew, J.M. \& Cole, S.T. The MycoBrowser portal: a comprehensive and manually annotated resource for mycobacterial genomes. Tuberculosis (Edinb) 91, 8-13 (2011).

63. Robinson, J.T. et al. Integrative genomics viewer. Nat Biotechnol 29, 24-26 (2011).

\section{ACKNOWLEDGEMENTS}

The following reagents were obtained through BEI Resources, NIAID, NIH: Monoclonal AntiMycobacterium tuberculosis GroEL2 (Gene Rv0440), Clone IT70 (DCA4) (produced in vitro), NR-13657; Genomic DNA from Mycobacterium tuberculosis, Strain H37Rv, NR-48669. We acknowledge the Centre for Core Biotechnology Services at the University of Leicester for support with containment level 3 experiments and analysis of mycobacterial proteins. The project was supported by the UK Biotechnology and Biological Sciences Research Council grants BB/K000330/1 and BB/P001513/1 (GVM); the French Infrastructure for Integrated Structural Biology (FRISBI) ANR-10-INBS-05 grant (MCG), the Ministry of Higher Education and Scientific Research Iraq (KA), and the Wellcome Trust 204538/Z/16/Z (SJW).

\section{AUTHOR CONTRIBUTIONS}

Conceptualization, OT, MCG, SJW and GVM; Methodology, CR, MW, AAW, $\mathrm{HOH}$; Investigation KA, OT, PB, HJ, ADV, SJW, GVM; Analysis, OT, KA, HJ, PB, AAW, SJW, MCG, GVM; Resources, ILB, MIV, and AAW; Writing -Original Draft, GVM, SJW, MCG; 
Writing - Review and Editing, GVM, SJW, MCG, ILB, OT, HOH. Funding Acquisition, GVM,

MCG, SJW, AK. Supervision, GVM, OT, SJW, HOH and MCG.

\section{DECLARATION OF INTERESTS}

Authors declare no competing interests.

\section{SUPPLEMENTARY TABLES}

Table S1. The transcriptional signature of PknB-depletion in M. tuberculosis, comparing pristinamycin-inducible PknB complemented strains in sucrose magnesium medium with and without pristinamycin.

A separate excel file

*Significantly differentially expressed genes were identified using a moderated t-test ( $p$-value $<0.05$ with Benjamini and Hochberg multiple testing correction) and fold change $>1.8$ from three biological replicates. 65 genes were significantly induced and 34 genes were repressed by PknB-depletion in replicating M. tuberculosis.

Table S3. The Lsr2 regulon.

A separate excel file

**Putative regulons for wild type Lsr2 mapped to the $M$. tuberculosis H37Rv genome (Mycobrowser release 3 2018-06-05, ${ }^{62}$ ). Individual ChIP-seq replicates are shown; the Lsr2 regulon was defined to have binding events in 2 of 3 biological replicates. Genes were included in the predicted regulon if the Lsr2 binding site was immediately upstream of, or within, the coding sequence of each gene. For Lsr2 intergenic binding between divergent genes, both genes were reported. For Lsr2 intergenic binding between convergent genes, neither gene was reported.

Table S4. Genes directly impacted by additional Lsr2 binding events in phosphoablative (T112A) Lsr2 M. tuberculosis compared to wild type Lsr2 $M$. tuberculosis.

A separate excel file

${ }^{* * *}$ Genes were included in the predicted regulon if the Lsr2 binding site was immediately upstream of, or within, the coding sequence of each gene. For Lsr2 intergenic binding between divergent genes, both genes were reported. For Lsr2 intergenic binding between convergent genes, neither gene was reported. Annotation from Mycobrowser (Release 3 2018-06-05, ${ }^{62}$ ). 


\section{FIGURES}

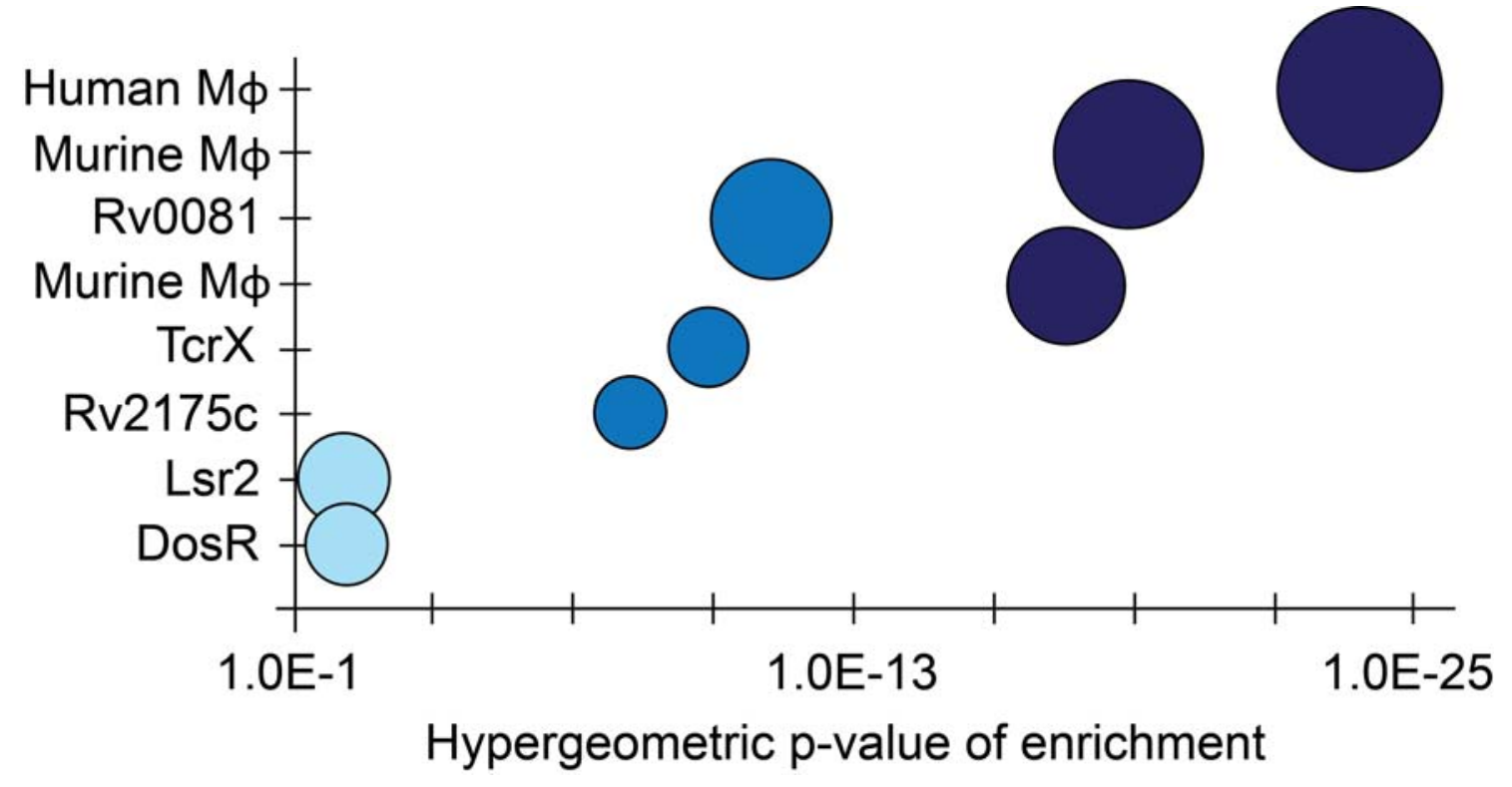

Figure 1. Transcriptional impact of PknB depletion in M. tuberculosis. The scheme highlights induced regulatory patterns from microarray (navy), transcription factor overexpression (blue), and ChIP-seq (light blue) studies referenced in the text. Enrichment $\mathrm{p}$-value plotted on $\mathrm{x}$-axis; bubble size proportional to number of overlapping genes in the signatures. $\mathrm{P}<0.02$ is considered as statistically significant. 


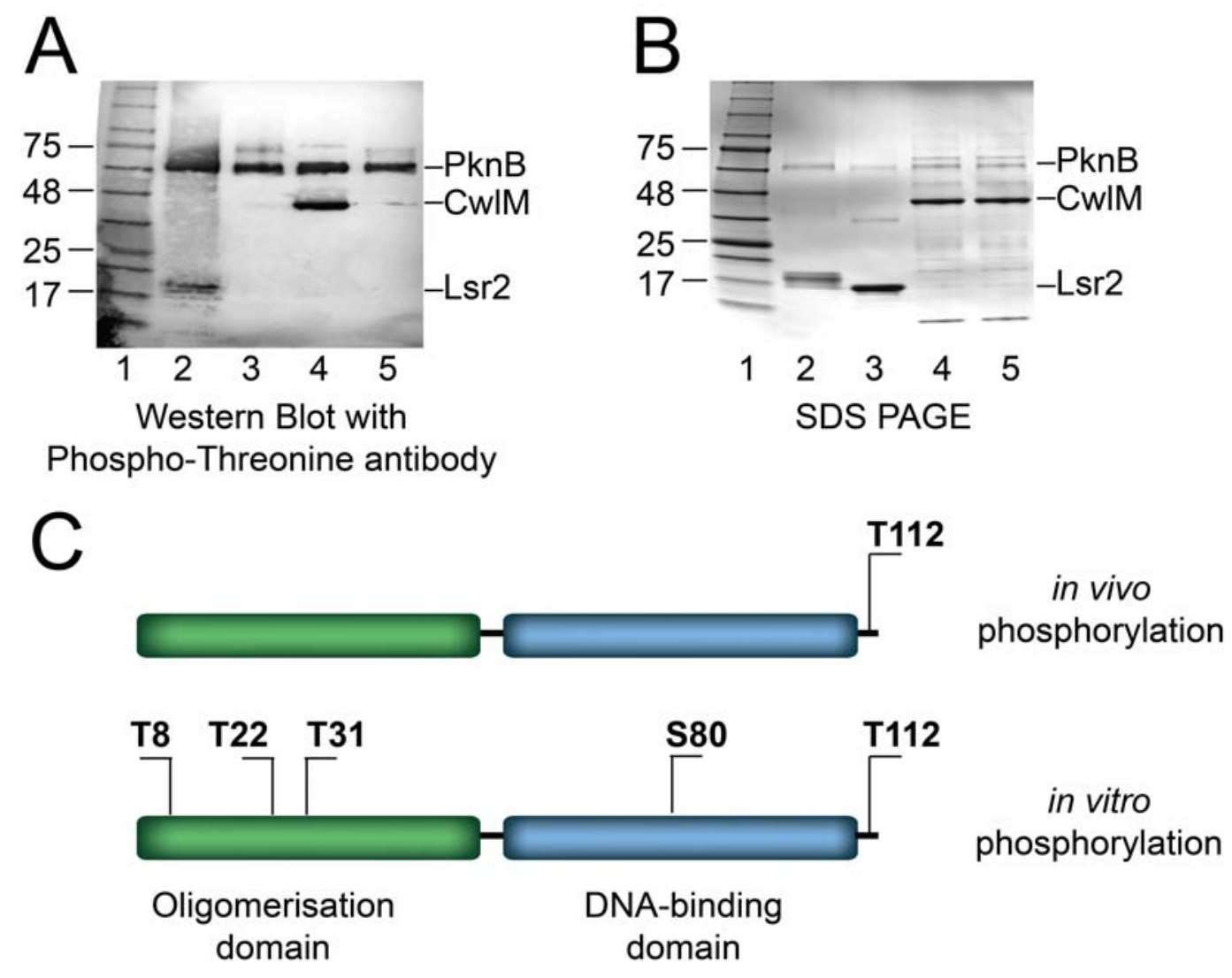

\begin{tabular}{|c|c|c|c|}
\hline Peptide & $\begin{array}{l}\text { Phospho } \\
\text { residue }\end{array}$ & $\begin{array}{l}\text { Observed } \\
\text { mass/ } \\
\text { charge }\end{array}$ & $\begin{array}{l}\text { Actual } \\
\text { mass }\end{array}$ \\
\hline KVTVRLVDDFDGSGAADETVEFGLDGVTYEIDLSTK & T8 & $1296.28(3)$ & 3585.82 \\
\hline KVTVTLVDDFDGSGAADETVEFGLDGVTYEIDLSTK & T22 & $1296.28(3)$ & 3585.82 \\
\hline GLDGVTYEIDLSTK & T31 & $795.87(2)$ & 1589.72 \\
\hline GAIDREQSAAIR & S80 & $683.83(2)$ & 1365.64 \\
\hline IPADVIDAYHAAT & T112 & $718.83(2)$ & 1435.64 \\
\hline
\end{tabular}

Figure 2. Identification of Lsr2 as a substrate of PknB. (A) Recombinant Lsr2 was phosphorylated by the recombinant enzymatic domain of PknB. Phosphorylated proteins were detected by western blot using a phospho-threonine antibody. Recombinant CwIM was used as positive control. 1- protein markers; 2 - Lsr2 incubated with PknB and ATP; 3 - Lsr2 incubated with PknB without ATP; 4 - CwIM incubated with PknB and ATP; 5 - CwIM incubated with PknB without ATP (B) SDS-PAGE revealed a shift in Lsr2 mobility upon phosphorylation (lanes identical to panel A). (C) Schematic presentation of phosphosites identified in phopshoproteomics studies (top) ${ }^{5}$, and in vitro (bottom). Lsr2 phosphorylation at T112- was 2.54-fold higher in PknB-producing M. tuberculosis compared to PknB-depleted M. tuberculosis (D) Phosphopeptides detected by mass-spectrometry; phosphorylated residues shown in red font. 


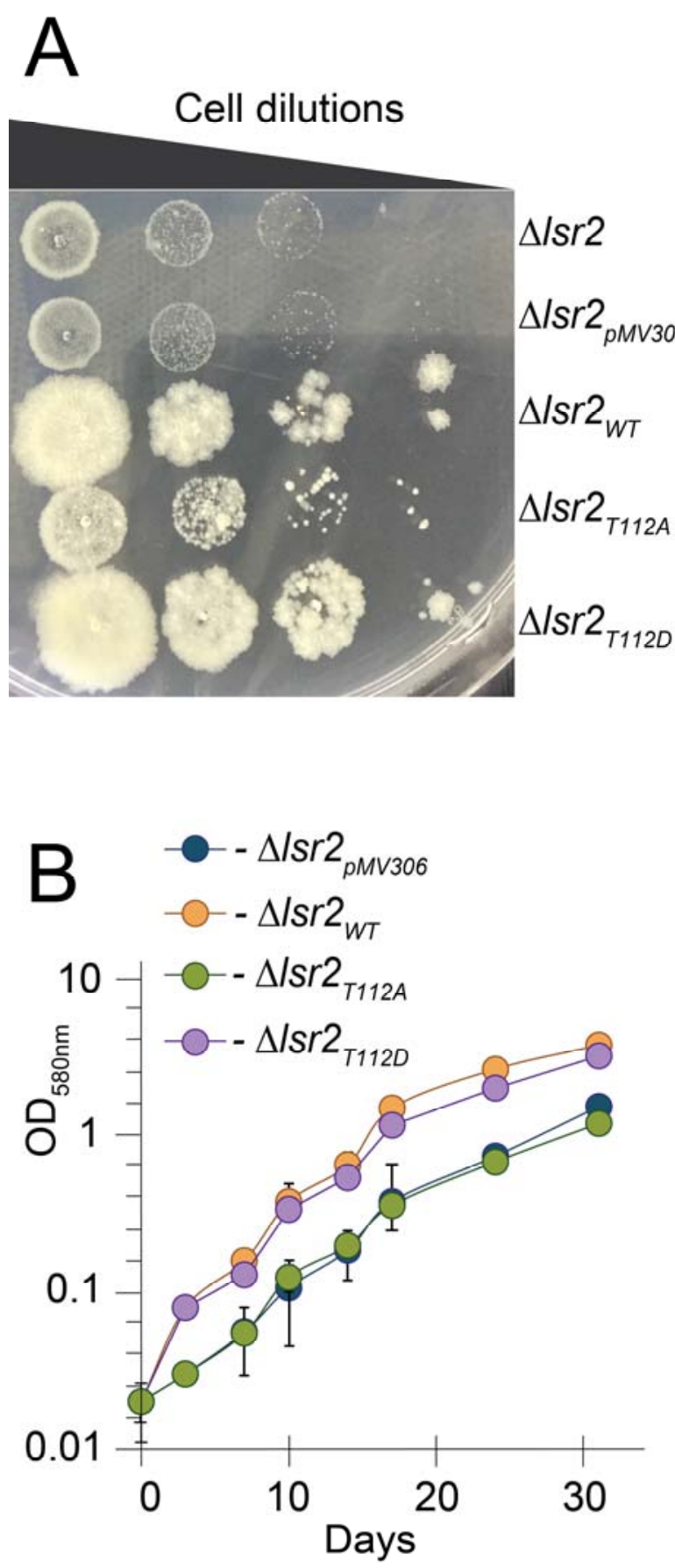

\section{C}

Western Blot with anti-GroEL \& anti-Lsr2

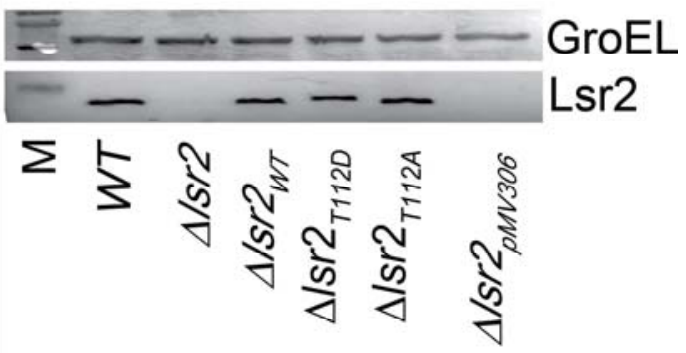

D

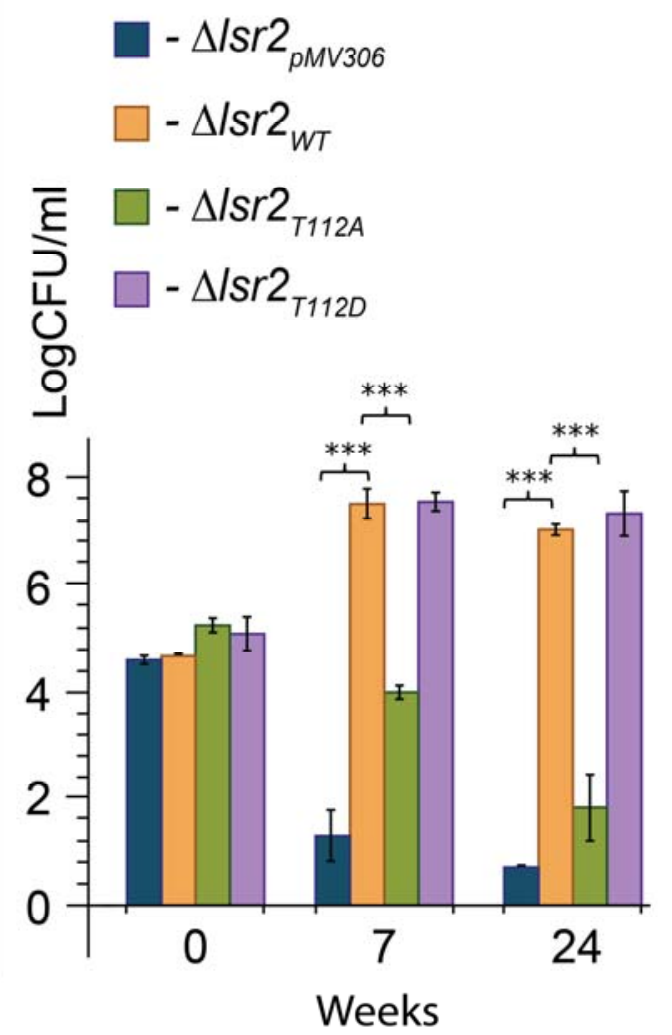

Figure 3. Phosphoablative T112A variant does not complement growth and survival defect of Isr2 deletion mutant. Growth of Isr2 deletion mutant expressing wild-type, T112A or T112D variants was compared with growth of the deletion mutant containing the empty vector. (A) 7H10 agar; (B) $7 \mathrm{H} 9$ liquid medium without shaking. Data presented as mean \pm SEM $(\mathrm{N}=6)$. (C) Expression of $L s r 2$ and $L s r 2$ variants from pMV306 was verified by Western blotting of M. tuberculosis lysates using an anti-Lsr2 antibody. Expression of GroEL was used as loading control. (D) T112A mutation impairs $M$. tuberculosis survival in the Wayne model of non-replicating persistence. M. tuberculosis Lsr2 mutants were incubated in sealed tubes with gentle mixing for up to 24 weeks. Data presented as mean $\pm S E M(N=6)$.

${ }^{* * *}$ Statistically different in $\Delta / s r 2_{p M V}$ or $\Delta / s r 2_{T 112 A}$ compared with $\Delta / s r 2_{W T}$ and $\Delta / s r 2_{T 112 D}$ $(p<0.001)$ 

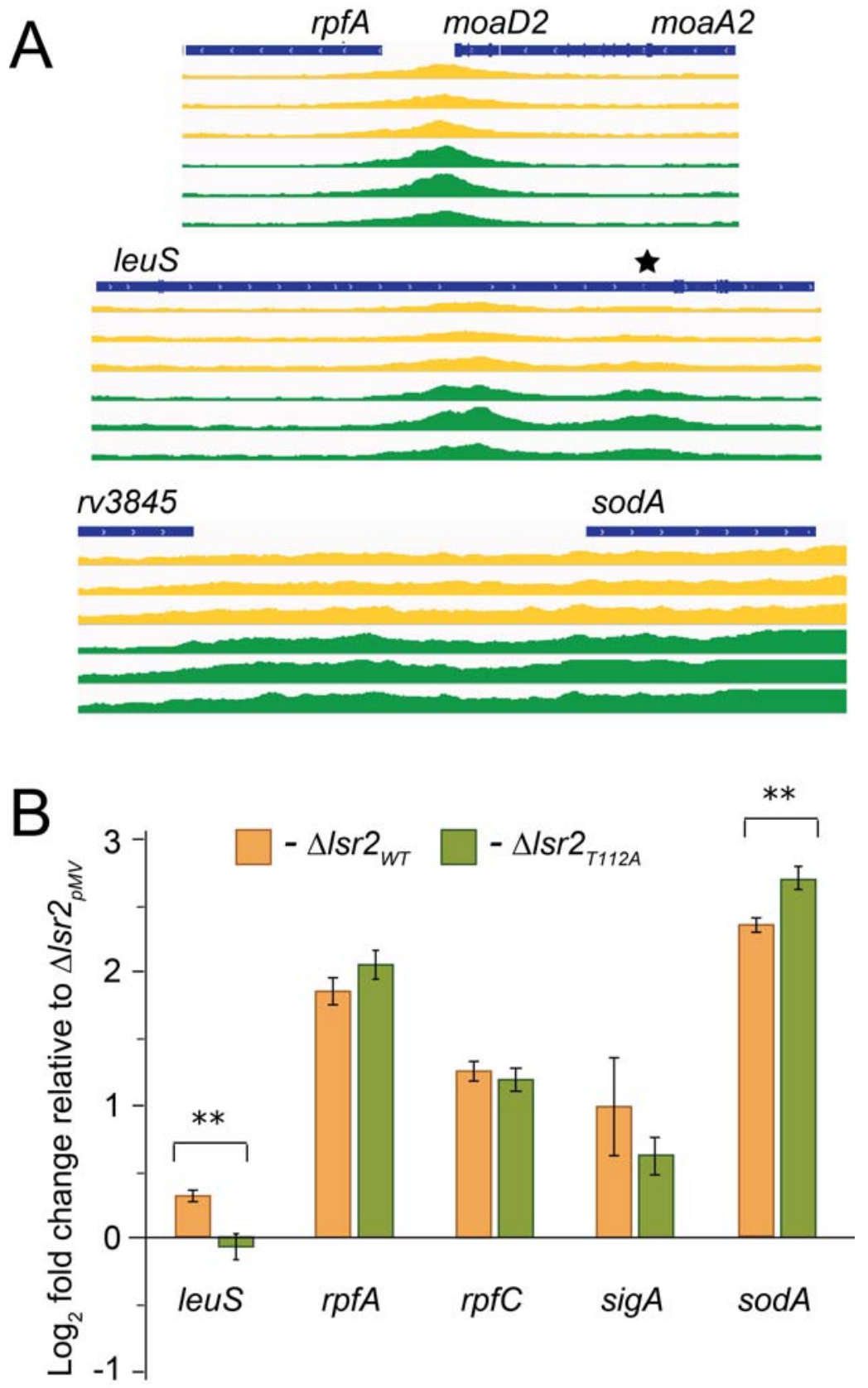

Figure 4. T112A mutation alters Lsr2 binding to DNA and gene expression patterns.

(A) Representative plots describe Lsr2 binding upstream of $r p f A$, intragenic binding in leuS, or both intergenic and intragenic binding in $\operatorname{sod} A$, showing greater Lsr2 binding in three biological replicates of phosphoablative $\Delta / s r 2_{T 112 A}$ (green) compared to $\Delta / s r 2_{W T}$ (yellow). A black asterisk marks the position of a Lsr2 binding site in leuS. Plots adapted from Integrative Genomics Viewer (IGV, ${ }^{63}$ ). Binding patterns in $\Delta / s r 2_{T 112 D}$ were identical to those in $\Delta / s r 2_{W T}$ and not shown for clarity. (B) Expression of leuS, rpfA, rpfC, sigA and $\operatorname{sod} A$ relative to $\Delta / s r 2_{p M V 306}$ measured by quantitative RT-PCR in $\Delta / s r 2_{p M V 306}, \Delta / s r 2_{W T}, \Delta / s r 2_{T 112 A}$ and normalised to $16 s r R N A$ and $\Delta / s r 2_{p M V 306}$. Data presented as mean $\pm S E M(N=6)$.

${ }^{*}$ Statistically different in $\Delta / s r 2_{T 112 A}$ compared with $\Delta / s r{ }_{W T}(\mathrm{p}<0.01)$. 

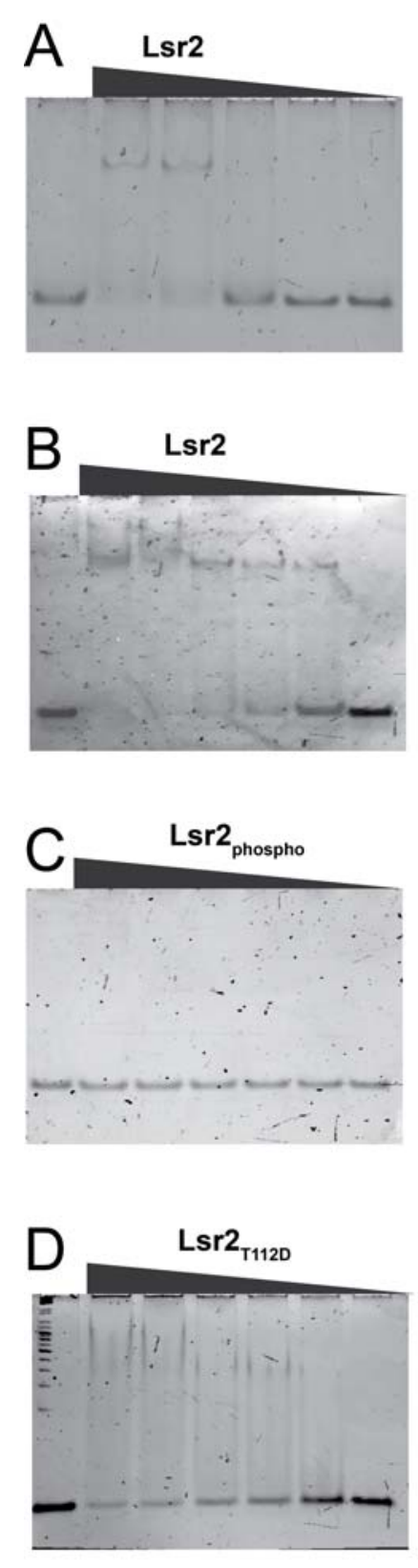
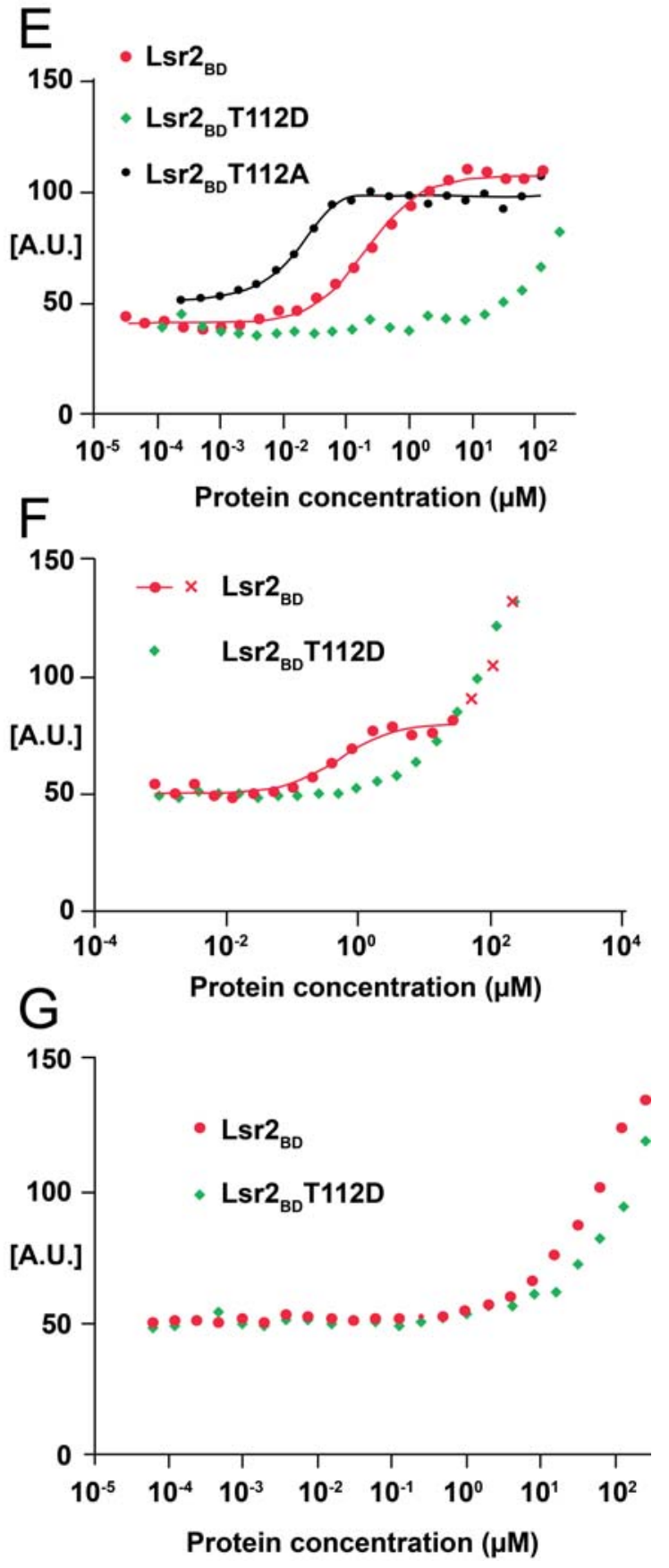

Figure 5. Phosphorylation or T112D mutation prevent Lsr2 binding to DNA. (A) Fulllength Lsr2 was mixed with leuS fragment; (B) Full-length Lsr2 was mixed with the mutated leuS $S_{\mathrm{MUT}}$ fragment; (C) Full-length Lsr2 was phosphorylated by PknB and mixed with leuS fragment; (D) Full-length T112D Lsr2 was mixed with leuS fragment. Lsr2 was added to DNA at a range of concentrations $(0.95-7.6 \mu \mathrm{M})$. Representative results from three independent experiments shown. (E-G) Quantification of Lsr2 ${ }_{\mathrm{BD}}$ (DNA binding domain) interaction with DNA by fluorescence anisotropy. Titration of 5' Alexa Fluor 488 DNA (4 nM) by Lsr2 $2_{\mathrm{BD}}$ WT (red), Lsr2 ${ }_{\mathrm{BD}}$ T112A (black) and Lsr2 ${ }_{\mathrm{BD}}$ T112D (green). (E) the previously identified DNA sequence (CGCGCATATATGCG); $(\mathrm{F})$ leuS binding site (AATTCGGCAAAATCGGTAAG), red crosses designate points that were not used for Kd calculations; (G) mutated leuS binding site (AACTCGGCGAGGTCGGTCAG). 

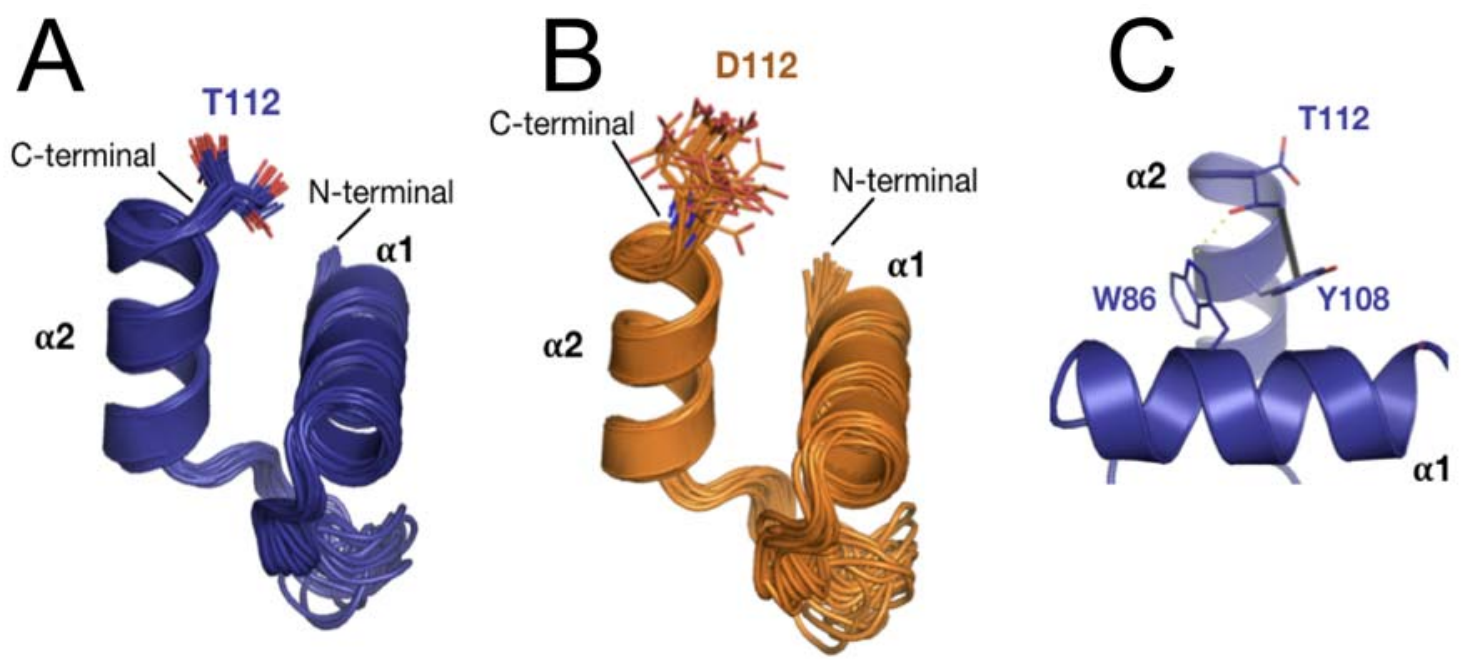

Figure 6. Solution structure of $\operatorname{Lsr}_{\mathrm{BD}}(\mathrm{A})$ and $\mathrm{Lsr}_{\mathrm{BD}} \mathrm{T}_{112 \mathrm{D}}(\mathrm{B})$. Superimposition of the 20 best calculated structures in cartoon representation with the last residues represented as sticks $(A$ and $B)$. In Lsr2 ${ }_{B D}$ the threonine $T 112$ side chain interacts with both tyrosine Y108 $(3.7 \AA)$ and tryptophan W86 (3.0 $)$, all represented in sticks $(C)$. 


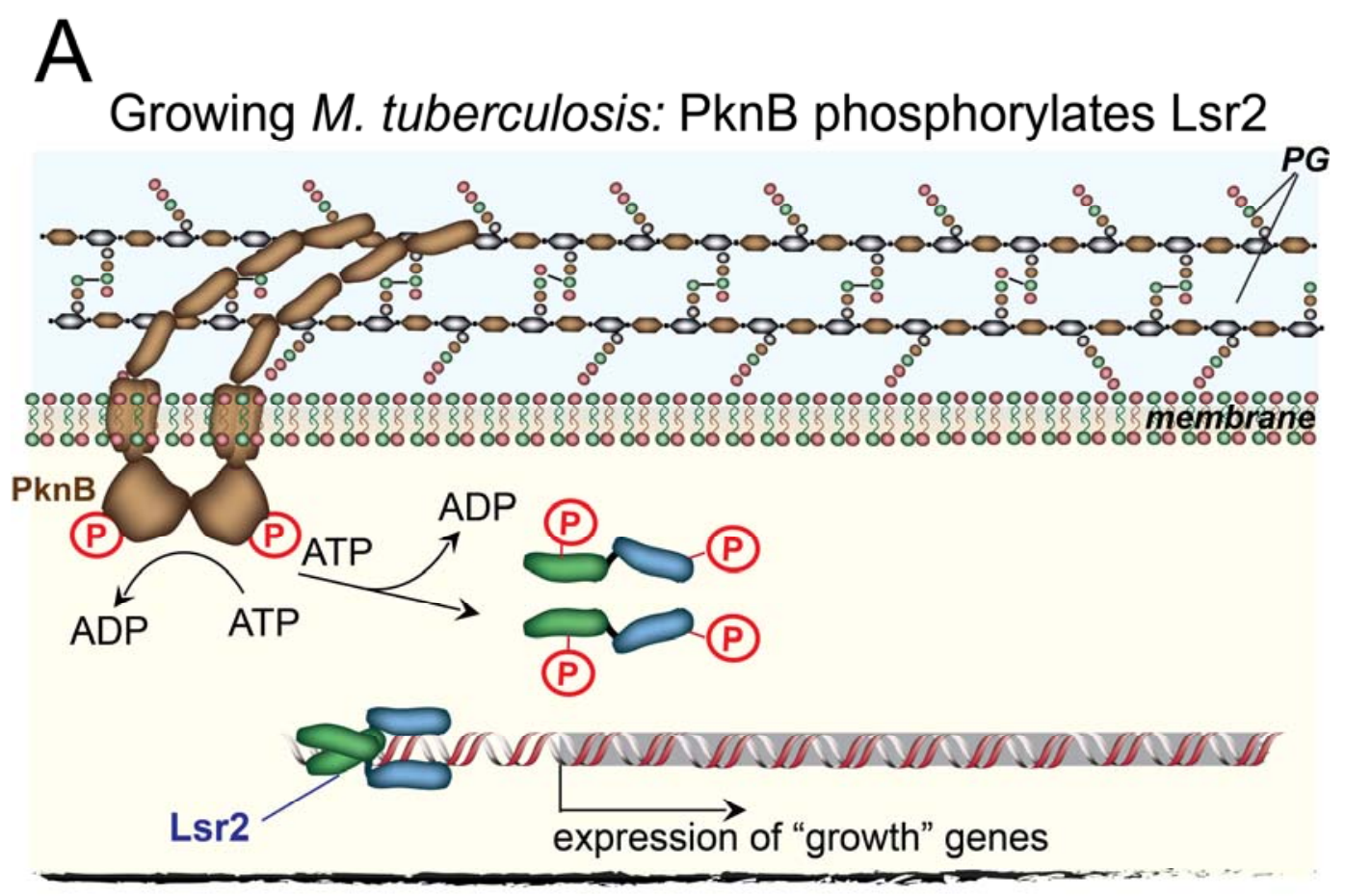

\section{B}

Non-growing M. tuberculosis: Lsr2 is not phosphorylated

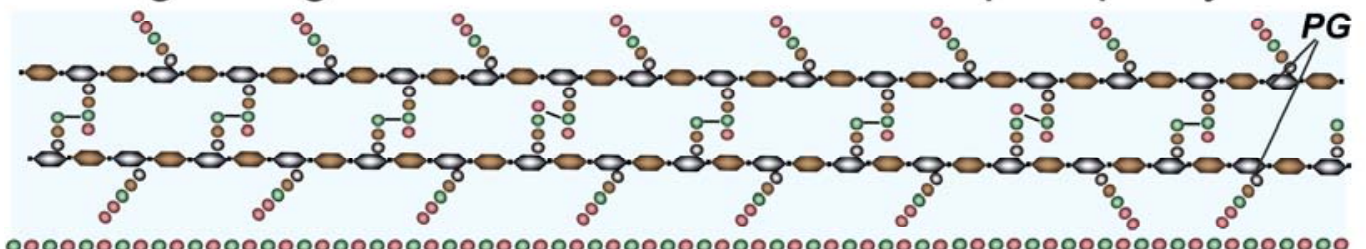

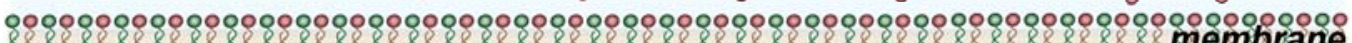
0000000000000000000000000000000000000000000000000000000000000000000000000

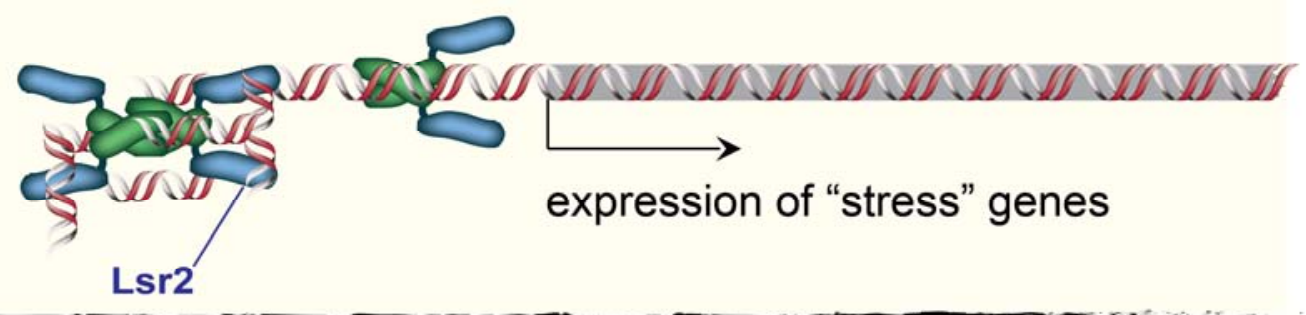

Figure 7. Lsr2 phosphorylation controls DNA binding and gene expression. (A) During growth PknB-mediated phosphorylation tunes gene expression to optimal levels and prevents activation of pathways disadvantageous for growth. Lsr2 is present as a mixture of phosphorylated and non-phosphorylated forms. The amount of phosphorylated Lsr2 is determined by activity of PknB, which is active during growth. (B) During non-permissive growth conditions, PknB is not produced and T112-nonphosphorylated Lsr2 binds DNA to a greater extent, switching on alternative transcriptional pathways and stress responses. 